\title{
Nano-encapsulated tryptanthrin derivative for combined anticancer therapy via inhibiting indoleamine 2,3-dioxygenase and inducing immunogenic cell death
}

\author{
Canyu Yang ${ }^{1}$ Bing He${ }^{1}$, Qiang Zheng ${ }^{2}$ Dakuan Wang ${ }^{1}$, Mengmeng Qin ${ }^{1}$, Hua Zhang ${ }^{1}$, \\ Wenbing Dai ${ }^{1}$, Qiang Zhang ${ }^{1,3}$, Xiangbao Meng**,2 \& Xueqing Wang*,1 \\ ${ }^{1}$ Beijing Key Laboratory of Molecular Pharmaceutics, New Drug Delivery Systems, School of Pharmaceutical Sciences, Peking \\ University, Beijing 100191, PR China \\ ${ }^{2}$ State Key Laboratory of Natural \& Biomimetic Drugs, Department of Chemical Biology, School of Pharmaceutical Sciences, Peking \\ University, Beijing 100191, PR China \\ ${ }^{3}$ State Key Laboratory of Natural \& Biomimetic Drugs, Peking University, Beijing 100191, PR China \\ *Author for correspondence: wangxq@bjmu.edu.cn \\ **Author for correspondence: xbmeng@bjmu.edu.cn
}

\begin{abstract}
Aim: We developed a polycaprolactone-based nanoparticle (NP) to encapsulate tryptanthrin derivative CY1-4 and evaluated its antitumor efficacy. Materials \& methods: CY-1-4 NPs were prepared and evaluated for their cytotoxicity and associated mechanisms, indoleamine 2,3-dioxygenase (IDO)-inhibitory ability, immunogenic cell death (ICD)-inducing ability and antitumor efficacy. Results: CY-1-4 NPs were $123 \mathrm{~nm}$ in size. In vitro experiments indicated that they could both induce ICD and inhibit IDO. In vivo studies indicated that a medium dose reduced $58 \%$ of the tumor burden in a B16-F10-bearing mouse model, decreased IDO expression in tumor tissues and regulated lymphocytes subsets in spleen and tumors. Conclusion: CY-1-4 is a potential antitumor candidate that could act as a single agent with combined functions of IDO inhibition and ICD induction.
\end{abstract}

First draft submitted: 20 February 2019; Accepted for publication: 12 June 2019; Published online: 6 September 2019

Keywords: combination therapy $\bullet$ IDO inhibition $\bullet$ immunogenic cell death $\bullet$ melanoma $\bullet$ tryptanthrin derivative

Indoleamine 2,3-dioxygenase (IDO) is a crucial heme-containing enzyme involved in tryptophan (Trp) metabolism in the kynurenine (Kyn) pathway [1]. Recent research has shown that IDO is overexpressed in various tumor cells and neighboring immune cells [2]. The Kyn pathway can modulate the immune response in tumors, the activity of which is closely related to tumor immune tolerance and escape including T-cell anergy [3]. Therefore, IDO has become a crucial target for antitumor immunotherapy and some inhibitors of it, such as NLG919 and INCB024360, have entered clinical trials. Some tryptanthrin derivatives have been reported to have IDO-inhibitory activity [4]. Moreover, tryptanthrins have also been confirmed to have antitumor activity in many in vivo tumor cell lines [5]. At higher concentrations, some of them can cause dysfunction and eventual destruction of mitochondria in cells and can induce cell death in a caspase-dependent manner [6].

CY-1-4 is a tryptanthrin derivative that was initially reported to have high antimalarial parasite activity. It can bind to ferric iron in heme and block heme crystallization to kill malaria parasites; its $\mathrm{IC}_{50}$ with $\beta$-hemin was reported to be only $4 \mu \mathrm{M}$ [7]. The similarity of CY-1-4's antimalarial mechanism with that of chloroquine and artemisinin suggests that it probably exerts antitumor efficacy via a mitochondrion-mediated pathway. In addition, the high affinity to heme indicates that it could also interact with IDO. Nowadays, the combination of IDO inhibition and anticancer agents is under analysis in both preclinical and clinical studies. On the one hand, IDO expression might be spontaneously upregulated in antitumoral responses induced by an established method of chemotherapy or radiotherapy [8]. Such mechanisms could dampen the effectiveness of treatment. So, IDO inhibition has been reported to improve the response to chemotherapeutic agents in a mouse breast cancer model [9]. On the other hand, cell death caused by chemotherapeutic agents may be immunogenic, and immunogenic cell death (ICD) 


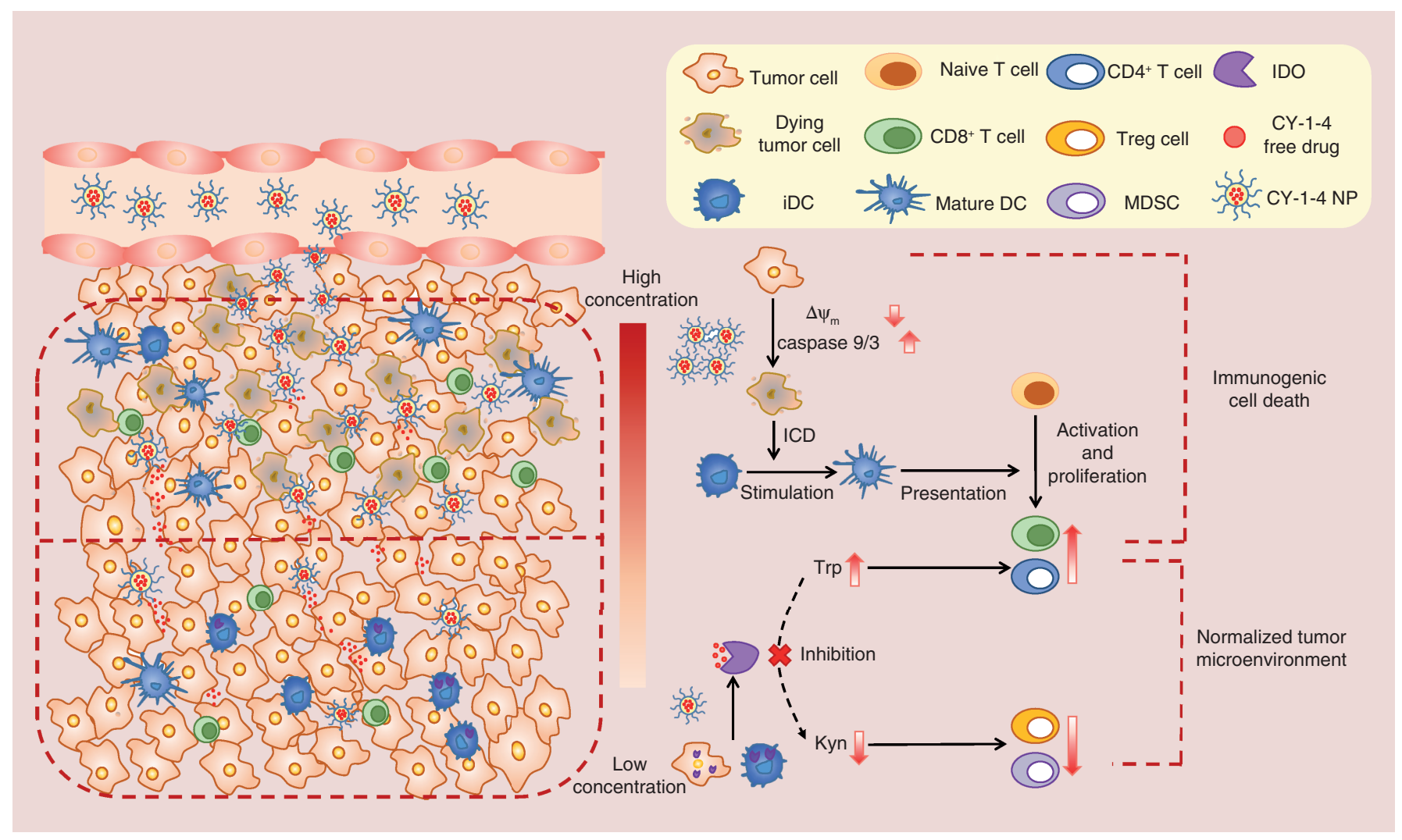

Figure 1. Schematic representation of CY-1-4 nanoparticles playing bifunctional roles in triggering immunogenic cell death and decreasing indoleamine 2,3-dioxygenase activity in a concentration-dependent manner.

would in turn potentiate antitumor immune response [10]. Against this background, a potentially good approach for inhibiting tumor growth would be to use a compound such as CY-1-4 possessing both of these two functions.

In this study, we investigated the antitumor ability of CY-1-4 to induce cell death via a mitochondrial pathway and IDO-inhibitory activity. Due to its poor solubility, we constructed a nanoparticle (NP) delivery system to encapsulate CY-1-4 (CY-1-4 NPs). By establishing this delivery system, we hypothesized that CY-1-4 NPs could perform the following: improve the solubility; achieve an enhanced permeability and retention (EPR) effect and accumulate more NPs in tumor tissues [11]. As depicted in Figure 1, CY-1-4 NPs could kill cancer cells by mitochondrion-mediated cytotoxicity and heme-blocking effects on both tumor cells and immune cells. In addition, CY-1-4 NPs could inhibit IDO activity in tumor tissues to restore T-cell survival and proliferation. They could also induce ICD to activate immature dendritic cells, thus promoting effector T-cell activation to attack and kill tumor cells. We investigated these functions and explored the relationship between dose and effect in tumor tissues. Our results indicated that CY-1-4 NPs could elicit dual functions in both chemotherapy and immunotherapy.

\section{Materials \& methods \\ Materials}

CY-1-4 (with molecular weight of 295.05) was previously synthesized by our group [12]. Polycaprolactone (PCL) and poly(ethyl ethylene phosphate)-co-poly( $\varepsilon$-caprolactone) $\left(\mathrm{mPEG}_{3000}-\mathrm{PCL}_{2500}\right)$ were both purchased from Advanced Polymer Materials Inc. (Montreal, Canada). All other materials were of analytical grade and were used without further purification.

\section{Cell lines \& cell culture}

The B16-F10 murine melanoma cell line and HeLa epithelial cervix adenocarcinoma cell line were provided by Cell Bank, Chinese Academy of Sciences (Shanghai, China). The cells were cultured in DMEM (M\&C) supplemented 
with $10 \%$ fetal bovine serum (FBS, Gemini Bio-products, CA, USA) and 1\% penicillin-streptomycin (M\&C) and maintained at $37^{\circ} \mathrm{C}$ in a humidified atmosphere containing $5 \% \mathrm{CO}_{2}$.

The DC 2.4 murine dendritic cell line and LX-2 human hepatic stellate cell line were also purchased from Cell Bank, Chinese Academy of Sciences. The cells were maintained in RPMI-1640 (M\&C) complemented with 10\% $\mathrm{FBS}$ and $1 \%$ penicillin-streptomycin at $37^{\circ} \mathrm{C}$ in a humidified environment with $5 \% \mathrm{CO}_{2}$.

\section{Animals}

Four- to six-week-old female C57BL/6 mice were purchased from Vital River Laboratory Animal Technology (Beijing, China; license number: SCXK [Jing] 2012-0001). All animal experiments were carried out in accordance with protocols approved by the Committee for Animal Research of Peking University and conformed to the Guide for the Care and Use of Laboratory Animals (NIH publication no. 86-23, revised 1985).

\section{Preparation \& characterization of CY-1-4-loaded nanoparticles}

CY-1-4-loaded NPs (CY-1-4 NPs) were prepared in accordance with the nanoprecipitation method [13]. Briefly, $375 \mu \mathrm{g}$ of CY-1-4 was dissolved in $0.75 \mathrm{ml}$ of a mixed solvent of acetone and ethyl alcohol (ratio = 9:1), and then $5.0 \mathrm{mg}$ of PCL and $0.5 \mathrm{mg}$ of $\mathrm{mPEG}_{3000}-\mathrm{PCL}_{2500}$ were also dissolved in the mixed solvent. The solution was added to $10 \mathrm{ml}$ of deionized water with $0.1 \mathrm{mg} / \mathrm{ml}$ Pluronic F68 (F68) dropwise; then, the samples were stirred in a water bath at $37^{\circ} \mathrm{C}$ for $15 \mathrm{~min}$. Finally, the rest of the organic solvent was removed with rotary evaporation at $37^{\circ} \mathrm{C}$ under a vacuum to obtain CY-1-4 NPs.

The CY-1-4 encapsulation efficiency was determined by HPLC (Agilent Technologies, CA, USA; mobile phase, acetonitrile:water = 1:1). The particle size of CY-1-4 NPs was analyzed by dynamic light scattering (Malvern Zetasizer Nano-ZS; Malvern, UK), and the morphology was observed through transmission electron microscopy (TEM, JEM-200CX; JEOL, Tokyo, Japan). The in vitro release of CY-1-4 from CY-1-4 NPs was evaluated by a dialysis method, under the same conditions as in the cytotoxicity experiments. Briefly, $1 \mathrm{ml}$ of CY-1-4 NPs was added into a dialysis bag (molecular weight cutoff $3500 \mathrm{Da}$ ) and then put into $10 \mathrm{ml}$ of blank culture medium. The stirring rate and temperature were kept at $600 \mathrm{rpm}$ and $37^{\circ} \mathrm{C}$, respectively. At predesigned time points, $100 \mu \mathrm{l}$ aliquots of culture medium were withdrawn and then immediately replaced with an equal volume of fresh culture medium. The released drug content was determined by HPLC.

\section{In vitro cytotoxicity assay}

A variety of cell lines including HeLa, B16-F10 and LX-2 cells were used to examine the cytotoxicity of CY-1-4 free drug and CY-1-4 NPs via the Cell Counting Kit-8 (CCK-8) test kit (Beyotime Biotech, Shanghai, China). CY-1-4 free drug was prepared by stepwisely diluting the stock solution of CY-1-4 (dissolved in DMSO at a concentration of $1 \mathrm{mg} / \mathrm{ml}$ ) with a blank culture medium. Moreover, classical chemotherapeutic drugs such as doxorubicin (Dox; Macklin, Shanghai, China), daunorubicin (Dau; Pharmabiology, USA) and 5-fluorouracil (5-FU; Macklin, Shanghai, China) were used on B16-F10 cells to give a comparison with CY-1-4 free drug regarding cytotoxicity. In brief, $5 \times 10^{3}$ cells per well were seeded in 96-well plates overnight. Then, the cell culture medium was removed and various concentrations of CY-1-4 free drug or CY-1-4 NPs were added to the cells. After $24 \mathrm{~h}$ of incubation and removing the remaining culture medium, $10 \mu \mathrm{l}$ of CCK-8 solution and $100 \mu \mathrm{l}$ of DMEM were added to each well of the plate and further incubated for another $2 \mathrm{~h}$. Absorbance was measured with a microplate reader at $492 \mathrm{~nm}$ (Multiskan FC; Thermo Scientific, MA, USA). Untreated wells were used as controls. Cell viability was calculated as follows: $\left(\mathrm{OD}_{\text {treated }}-\mathrm{OD}_{\text {blank }}\right) /\left(\mathrm{OD}_{\text {control }}-\mathrm{OD}_{\text {blank }}\right) \times 100 \%$.

\section{Mitochondrion-related cell death of CY-1-4 NPS}

Apoptosis analysis

The relative proportions of apoptotic cells and necrotic cells were assessed using Annexin V-FITC/propidium iodide (PI) apoptosis detection kit (Beyotime Biotech). B16-F10 cells were seeded in six-well plates at a density of $1 \times 10^{5}$ cells per well for $24 \mathrm{~h}$ before exposure to various concentrations of CY-1-4 NPs. After B16-F10 cells had been treated for another $24 \mathrm{~h}$, the cells were harvested, washed with cold phosphate-buffered saline (PBS; M\&C, Beijing, China) twice and prepared in a single-cell suspension. Apoptosis was measured using a flow cytometer (FACS Calibur system; BD Biosciences, Oxford, UK) with FITC-conjugated Annexin V and PI double staining. In accordance with the staining results, the cells could be divided into three groups: normal (Annexin V-and PI-negative), early apoptosis (Annexin V-positive and PI-negative) and late apoptosis/necrosis (Annexin V-and PI- 
positive). In this study, the early apoptotic cells and the late apoptotic/necrotic cells were subjected to quantitative analysis. Flow cytometry analysis was performed using FlowJo 6 software (Tree Star, OR, USA).

MMP analysis

The change of MMP was investigated to further understand the details of the mechanism of cell death. The alteration of the MMP was measured using Mitochondrial Membrane Potential Detection Kit (Beyotime Biotech), in accordance with the guidelines. B16-F10 cells were seeded in a six-well culture plate overnight and then treated with different concentrations of CY-1-4 NPs for $24 \mathrm{~h}$. After incubation with JC-1 fluorescent dye for $30 \mathrm{~min}$, the cells were washed and resuspended with JC-1 assay buffer. Flow cytometry analysis was performed using a flow cytometer (FACS Calibur system) and FlowJo 6 software.

Caspase-3 \& caspase-9 activity analysis

Caspase- 9 and caspase-3 activities were analyzed after treatment with different concentrations of CY-1-4 NPs using Caspase-9 Activity Assay Kit and Caspase-3 Activity Assay Kit (both from Beyotime Biotech). B16-F10 cells were seeded in 12-well plates for culture overnight and then treated with CY-1-4 NPs $(0-1 \mu \mathrm{M})$ for $24 \mathrm{~h}$. Activities of caspase- 9 and caspase- 3 were determined by incubating $50 \mu \mathrm{g}$ of total proteins per well with Ac-DEVD-pNA or Ac-LEHD-pNA substrates to develop a yellowish color. The absorbance of each well was measured with a microplate reader at $405 \mathrm{~nm}$.

ICD caused by $C Y-1-4 N P S$

HMGB1 release assay

HMGB1 distribution was tested using immunofluorescence analysis. Briefly, B16-F10 cells were seeded on a live cell imaging glass-bottomed dish at a density of $2 \times 10^{4}$ cells per well overnight and then treated with different drug formulations for $24 \mathrm{~h}$ (CY-1-4 NPs at $0.75 \mu \mathrm{M}$ and CY-1-4 free drug at $0.75 \mu \mathrm{M})$. Next, the intracellular HMGB1 was stained with antibodies in accordance with the immunofluorescence instructions. Briefly, cells were fixed with $4 \%$ paraformaldehyde for 20 min and permeabilized with $0.1 \%$ Triton X-100 for 10 min. Nonspecific binding sites were blocked by preincubation with $5 \%$ FBS in PBS for $30 \mathrm{~min}$, followed by incubation with primary antibody (Anti-HMGB1 antibody [EPR3507] ab79823; Abcam, Oxford, UK) overnight and then incubated with an Alexa Fluor 488-conjugated secondary antibody (Bioss, Beijing, China) for $2 \mathrm{~h}$ after three washes with PBS. Finally, the cells were stained with Hoechst 33258 (Solarbio, Beijing, China) and examined using a confocal laser scanning microscope (TCS SP5; Leica, Wetzlar, Germany).

Calreticulin exposure assay

The surface exposure of calreticulin (CRT) was examined by flow cytometry. B16-F10 cells were seeded into a 24-well plate at a density of $2 \times 10^{4}$ cells per well for growth overnight. After treatment with different drug formulations for $8 \mathrm{~h}$ (CY-1-4 NPs at $0.75 \mu \mathrm{M}$ and CY-1-4 free drug at $0.75 \mu \mathrm{M})$, the cells were harvested, washed twice with cold PBS and then resuspended in approximately $1 \times 10^{6}$ cells $/ \mathrm{ml}$ in ice-cold PBS. The cells were then incubated with primary antibody (anti-CRT antibody [EPR3924], ab92516; Abcam, Oxford, UK) for 30 min at room temperature in the dark. The cells were washed again and incubated with Alexa Fluor 488-conjugated secondary antibody for 30 min. Finally, the cells were washed in PBS and then analyzed by flow cytometry (FACS Calibur system).

DC stimulation \& activation effects

To evaluate the stimulation and activation effects of dendritic cells (DCs) using treated tumor cells, a coculture system was established. First, B16-F10 cells were seeded in six-well plates for growth overnight and then treated with different concentrations of CY-1-4 NPs for $24 \mathrm{~h}$. The culture medium (including cell debris) was then harvested and cocultured with DC 2.4 cells in another six-well plate for $24 \mathrm{~h}$. Finally, DC 2.4 cells were harvested to monitor their mature phenotypes by flow cytometry. The following monoclonal antibodies were used: APC-conjugated CD86 (105011), PE-conjugated CD80 (104707) and PE/Cy7-conjugated CD11c (117317; all purchased from Biolegend, CA, USA). Corresponding isotype antibodies were used as controls. The rates of positivity for CD80, CD86 and CD11c were used to represent the extent of DC activation [14,15].

Moreover, to validate that the effects of DC activation were from the dying cell debris and not from CY-1-4 NP stimulation, DC 2.4 cells were directly treated with different concentrations of CY-1-4 NPs for $24 \mathrm{~h}$, and the same phenotypes of CD11c, CD80 and CD86 were tested. The cytotoxicity of DC 2.4 cells was also evaluated. The DC 
2.4 cells were seeded in 96-well plates at 5000 cells per well overnight, and the CY-1-4 NP concentrations were $0.125,0.25,0.375,0.6,0.75,1$ and $3 \mu \mathrm{M}$. After incubation for $24 \mathrm{~h}$, cell viability was estimated by the CCK-8 test.

\section{In vitro IDO activity inhibition assay}

Enzymatic inhibition efficacy of CY-1-4 free drug on IDO

IDO catalyzes the oxidative cleavage of the pyrrole ring of the indole nucleus of tryptophan to yield $N$ formylkynurenine. The assays were performed at room temperature as described in the literature [16] using $20 \mathrm{nM}$ IDO (\#H00003620-P01; Novus Biologicals, CO, USA) and $2 \mathrm{mM}$ D-Trp in the presence of $20 \mathrm{mM}$ ascorbate, $3.5 \mu \mathrm{M}$ methylene blue and $0.2 \mathrm{mg} / \mathrm{ml}$ catalase in $50 \mathrm{mM}$ potassium phosphate buffer $(\mathrm{pH}$ 6.5). The initial reaction rates were recorded by continuously following the absorbance increase at $321 \mathrm{~nm}$ due to the formation of $N$-formylkynurenine. The $\mathrm{IC}_{50}$ values were calculated using nonlinear regression with normalized dose-response fit.

Cell-based IDO-inhibitory efficacy of CY-1-4 free drug \& CY-1-4 NPs

IDO was reported to be upregulated in various tumor cell lines after IFN- $\gamma$ stimulation [17], and the overexpression of IDO could be tested by the increase of kynurenine in the culture medium. Here, B16-F10 cells and HeLa cells were chosen to examine the IDO upregulation ability using colorimetric assay [18] and HPLC detection [19]. Briefly, cells were seeded in a 96-well plate at a density of $1 \times 10^{4}$ cells per well and allowed to grow overnight. Human recombinant IFN- $\gamma$ for HeLa cells and murine IFN- $\gamma$ for B16-F10 cells (both from PeproTech, NJ, USA) were then added to each well at a final concentration of $70 \mathrm{ng} / \mathrm{ml}$. For colorimetric assay, after $48 \mathrm{~h}$ of incubation, $150 \mu \mathrm{l}$ of supernatant per well was transferred to a new 96-well plate. $75 \mu \mathrm{l}$ of $30 \%$ trichloroacetic acid was added to each well and the mixture was incubated at $50^{\circ} \mathrm{C}$ for $30 \mathrm{~min}$ to hydrolyze $N$-formylkynurenine to kynurenine. For the colorimetric assay, supernatants were transferred to a new 96-well plate, mixed with an equal volume of Ehrlich reagent (2\% p-dimethylamino-benzaldehyde $\mathrm{w} / \mathrm{v}$ in glacial acetic acid) and incubated for $10 \mathrm{~min}$ at room temperature. Reaction product was measured at $490 \mathrm{~nm}$ by a plate reader. For HPLC analysis, the supernatant in each well was collected and centrifuged at $8000 \mathrm{rpm}$ for $10 \mathrm{~min}$ to remove dead cells. A total of $100 \mu \mathrm{l}$ of the supernatants per well was sampled for the kynurenine assay. The mobile phase for HPLC was 15 mM sodium acetate $(\mathrm{pH} 4.0)$ :acetonitrile $=92 \%: 8 \%$.

Then, the IDO-inhibitory effects of CY-1-4 and CY-1-4 NPs were tested with the optimized cell line. After various concentrations of CY-1-4 and CY-1-4 NPs had been added to the cells and incubated for 48 h, the supernatant in each well was analyzed by the HPLC as described above.

\section{In vivo therapeutic efficacy and safety experiment}

First, we compared the tumor inhibition efficacy and toxicity between CY-1-4 NPs and CY-1-4 free drug at different doses. Female C57BL6 mice at 4-6 weeks old were subcutaneously inoculated with B16-F10 tumor cells on the right side of the upper armpit $\left(1 \times 10^{6}\right.$ cells per mouse $)$. The mice were randomly distributed into seven groups when the tumor volume reached approximately $100 \mathrm{~mm}^{3}$ and treated with a high, medium or low dose of CY-1-4 NPs $(450,300$ or $150 \mu \mathrm{g} / \mathrm{kg})$ and free drug at the same doses every 2 days six-times via tail vein injection. CY-1-4 free drug was solubilized in DMSO at a concentration of $10 \mathrm{mg} / \mathrm{ml}$ and then diluted with PBS to the same concentrations as CY-1-4 NPs. The negative control group was treated with PBS every 2 days in the same way. Tumor sizes were measured every 2 days in two dimensions using calipers, and tumor volumes were calculated from the following formula: $\mathrm{V}=\left(\mathrm{A} \times \mathrm{B}^{2}\right) / 2$ ( $\mathrm{A}$ and $\mathrm{B}$ are the long and short diameters of the tumor). Mice were sacrificed on the 14th day. The organs of heart, liver, spleen, lung and kidney were kept for histological evaluation with hematoxylin and eosin (H\&E), and the tumor tissues were harvested and weighed immediately; tumor specimens were fixed with $4 \%$ paraformaldehyde and embedded in paraffin wax for IDO expression and apoptosis analysis. The IDO expression was analyzed via IDO immunohistochemical staining (anti-IDO, clone 10.1, Cat. \#MAB5412; Chemicon, MA, USA), and tumor cell apoptosis was analyzed via terminal deoxynucleotide transferase (TDt)-mediated dUTP-biotin nick-end labelling (TUNEL) assay. Immunostained sections were first scanned and acquired using the Vectra 3.0 Automated Quantitative Pathology Imaging System (PerkinElmer, MA, USA); then, image files created by Vectra were analyzed using InForm 2.4.1 image analysis software. The percentage of positive cells was calculated as the number of DAB-positive cells divided by the total number of cells in the field. 
Table 1. Basic characterization of CY-1-4 nanoparticles ( $n=3$, mean \pm standard deviation).

\begin{tabular}{lllll} 
Particle size $(\mathrm{nm})$ & PDI & Zeta potential $(\mathrm{mV})$ & Encapsulation efficiency (\%) & Drug loading (\%) \\
\hline $123.7 \pm 2.97$ & $0.211 \pm 0.01$ & $-18 \pm 1.21$ & $88.55 \pm 2.13$ & $4.39 \pm 0.20$ \\
\hline PDI: Polydispersity Index. & & & &
\end{tabular}

Next, we selected a group of CY-1-4 NPs at the medium dose $(300 \mu \mathrm{g} / \mathrm{kg})$, which showed the best tumor inhibition efficacy in the experiment above, to investigate the IDO-related immunoregulatory function. 1-Methyltryptophan (1-MT), the classical IDO signal pathway inhibitor, which has been intensively studied [8], was chosen as a reference. Modeling methods in mice were the same as above. Briefly, mice were randomly divided into three groups ( $\mathrm{n}=8$ each group): one featured the administration of CY-1-4 NPs ( $300 \mu \mathrm{g} / \mathrm{kg}$ ) every 2 days five-times via tail vein injection, while one featured administration of the classic IDO inhibitor 1-MT $(200 \mathrm{mg} / \mathrm{kg})$ every day ten-times via intragastric administration. There was also a negative control group, which was treated with PBS every 2 days five-times via tail vein injection. Tumor sizes were measured in the same way as illustrated above. Mice were sacrificed on the 12th day. Their spleens were harvested for further examination of immune cells. Tumor tissues were harvested, weighed, photographed and subjected to immune cell population analysis.

Immune cell population analysis

The immune cell populations $\left(\mathrm{CD}^{+} \mathrm{T}\right.$ cells, $\mathrm{CD}^{+} \mathrm{T}$ cells, $\mathrm{CD}^{+} \mathrm{T}$ cells, regulatory $\mathrm{T}$ cells $\left[\mathrm{CD} 4{ }^{+} \mathrm{CD} 25^{+} \mathrm{FoxP}^{+}\right.$Tregs], myeloid-derived suppressive cells $\left.\left[\mathrm{CD} 11 \mathrm{~b}^{+} \mathrm{Gr}-1^{+} \mathrm{MDSCs}\right]\right)$ in spleens and tumor tissues with different treatments were analyzed by flow cytometry. Cell suspensions from spleens or tumor tissues were filtered with a nylon net $(90 \times 90 \mathrm{~mm}$; Dakewe Biotech, Shenzhen, China) and red blood cells were lysed with Red Blood Cell Lysis Buffer (Catalog \#5831-100; Biovision, CA, USA). The immune cells from tumor tissues were separated with a kit (WBC1092Z; HaoYang Biological, Tianjin, China).

For membrane staining, cells were incubated with combinations of antibodies (CD3, CD4, CD8, CD11b, Gr-1 and CD25). For intracellular staining for the antibody FoxP3, cells were fixed and permeabilized immediately after cell surface staining in accordance with the True-Nuclear ${ }^{\top M}$ Transcription Factor Staining Protocol (Biolegend). All antibodies were purchased from Biolegend and flow data were collected on a Gallios flow cytometer (Beckman Coulter, CA, USA). The data were analyzed using FlowJo 6 software (Tree Star).

\section{Statistical analysis \& formatting}

Data are expressed as mean \pm standard error of mean (SEM) or mean \pm standard deviation (SD). Differences between groups in terms of continuous variables were analyzed using Student's t-test. Analysis of variance (ANOVA) was performed to compare multiple groups. A value of $\mathrm{p}<0.05$ was considered statistically significant. All statistical analyses in this study were performed and formatted by GraphPad Prism 6.

\section{Results}

\section{Preparation \& characterization of CY-1-4 NPs}

In previous studies, nanotechnology was used to improve the solubility of hydrophobic compounds including tryptanthrin and its derivatives [20,21] and achieved combination therapies based on chemotherapy and IDO inhibition [22-24]. Moreover, nanocarriers were also shown to increase the immunogenicity of ICD inducers [10]. In this study, we chose PCL and mPEG-PCL, which were approved by the US FDA as a drug-carrying polymer matrix. Figure $2 \mathrm{~A} \& \mathrm{~B}$ presents the preparation process and chemical structure of CY-1-4. TEM images show that CY-1-4 NPs were well dispersed with a spherical morphology. The mean hydrodynamic diameter was about $123 \mathrm{~nm}$ and the polydispersity index (PDI) was relatively low, showing that the size was uniform (Table $1 \&$ Figure 2C). The encapsulation efficiency and drug-loading capacity ensured that the formulation could meet the requirements for the following experiments. The rate of release of CY-1-4 from CY-1-4 NPs under conditions the same as in the cytotoxicity experiments was lower than $6.6 \%$ at $24 \mathrm{~h}$, showing that the cytotoxicity mainly resulted from NPs, but not the released CY-1-4.

\section{CY-1-4 showed relatively high cytotoxicity in different tumor cells}

To evaluate its potential as a chemotherapy drug, the cytotoxicity of CY-1-4 free drug and CY-1-4 NPs at different concentrations was determined by CCK-8 tests in different cell lines. The CY-1-4 free drug was also tested in 


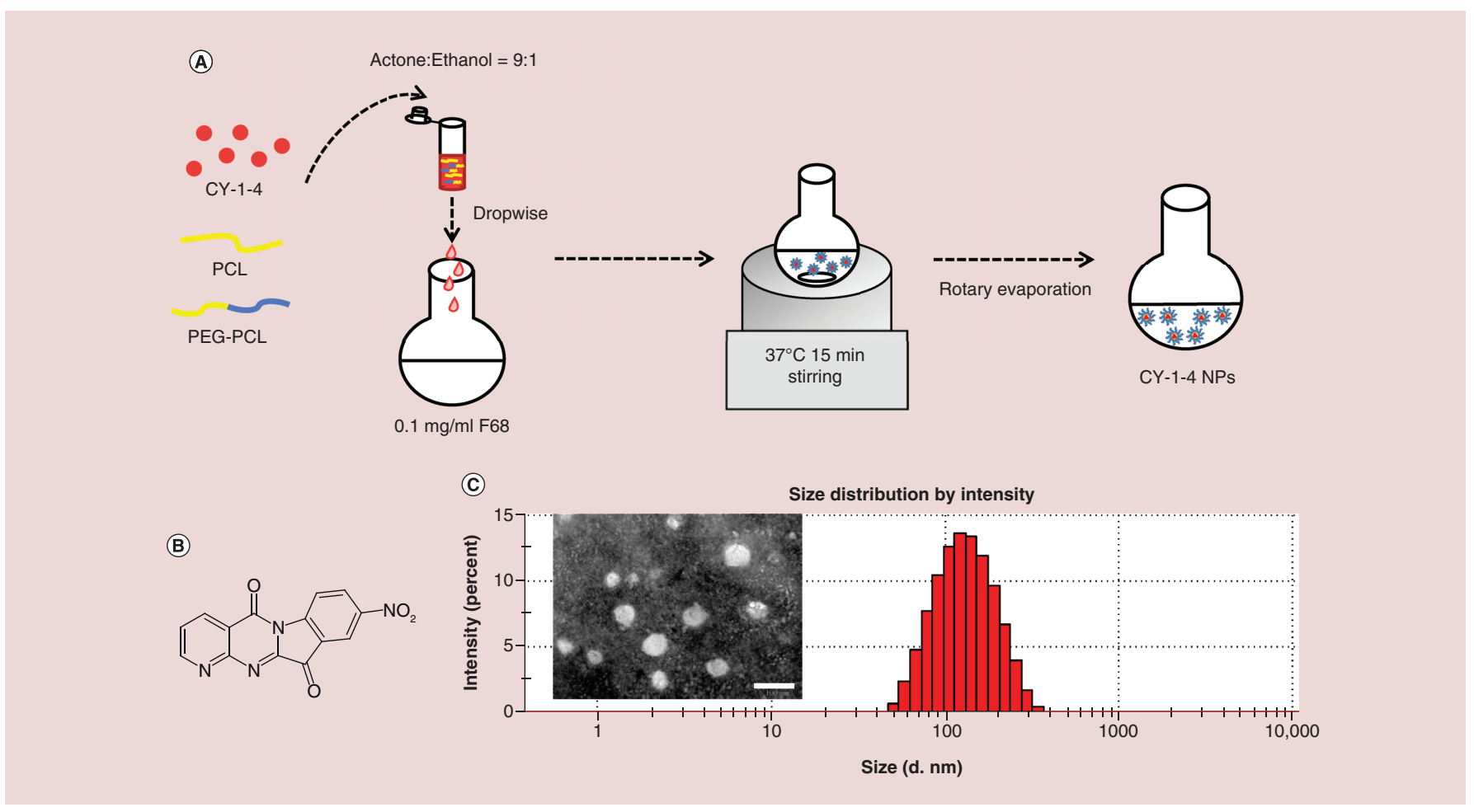

Figure 2. Preparation and physicochemical properties of CY-1-4 nanoparticles. (A) Preparation scheme of CY-1-4 NPs. (B) Chemical structure of CY-1-4. (C) Transmission electron microscopy images and particle size distribution of CY-1-4 NPs. Scale bar, $100 \mathrm{~nm}$. NP: Nanoparticle.

normal cells (LX-2) for comparison (Figure 3A). The $\mathrm{IC}_{50}$ value of CY-1-4 free drug was $0.49 \mu \mathrm{M}$ in B16-F10 cells and $0.34 \mu \mathrm{M}$ in HeLa cells. The $\mathrm{IC}_{50}$ of CY-1-4 NPs was $0.50 \mu \mathrm{M}$ in B16-F10 cells and $0.60 \mu \mathrm{M}$ in HeLa cells (Figure 3B). These findings indicated that HeLa cells were more sensitive to free drug than NPs at the same concentration. Notably, the cytotoxicity on LX-2 was half that on B16-F10 cells and HeLa cells, for which the $\mathrm{IC}_{50}$ reached $0.83 \mu \mathrm{M}$. Additionally, to compare the toxicity of CY-1-4 and traditional chemotherapy drugs, we also performed the same experiments using Dox, Dau and 5-FU on B16-F10 cells (Supplementary Figure 1). Dox and Dau caused less cell death than CY-1-4 (with $\mathrm{IC}_{50}$ of 0.89 and $1.09 \mu \mathrm{M}$, respectively), and 5-FU did not exhibit apparent cytotoxicity at the same concentration range.

\section{CY-1-4 NPs induced cell death in a mitochondrion-mediated pathway}

To further explore the apoptosis and necrosis with different concentrations of CY-1-4 NPs, we chose four concentrations $(5 \mathrm{nM}, 0.125,0.25$ and $1 \mu \mathrm{M})$ to examine the apoptosis and necrosis of B16-F10 cells via flow cytometry with Annexin V-FITC/PI fluorescence staining (Figure 3C). The results showed that the $1 \mu \mathrm{M}$ treatment of tumor cells generated a significant increase in cell death, which accounted for more than $80 \%$ of all cells, while the remaining three concentrations $(5 \mathrm{nM}, 0.125$ and $0.25 \mu \mathrm{M})$ did not show much difference in apparent apoptosis or necrosis.

As mentioned in the introduction, because of the similarity of antimalarial mechanism with chloroquine and artemisinin, the changes of mitochondrial membrane potential (MMP) and caspase- $9 / 3$ activities induced by CY1-4 NPs were examined to explore whether the cell death resulted from a change of mitochondria. Intracellularly, the shift of JC-1 from aggregates (red fluorescence) to monomers (green) indicated the decrease of MMP, which was a signal of early apoptosis of cells. MMP change could be observed in fluorescence photomicrographs at three different concentrations (Figure 3D), and the quantitative results of flow cytometry confirmed this (Figure 3E \& F). The JC-1 green percentages increased with higher CY-1-4 NP concentration, which indicated the decrease of MMP and the early apoptosis signal. The activity of caspase- 9 was crucial to mitochondrion-mediated apoptosis and the upregulated caspase- 9 activity indicated the initiation of apoptosis. Moreover, the caspase- 3 activity also showed the overall status of apoptosis. As Figure $3 \mathrm{G}$ depicts, the relative caspase- 9 activity was increased as the concentration 
(A)

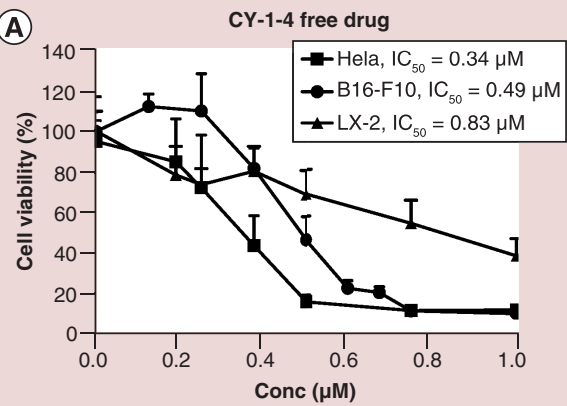

(B)

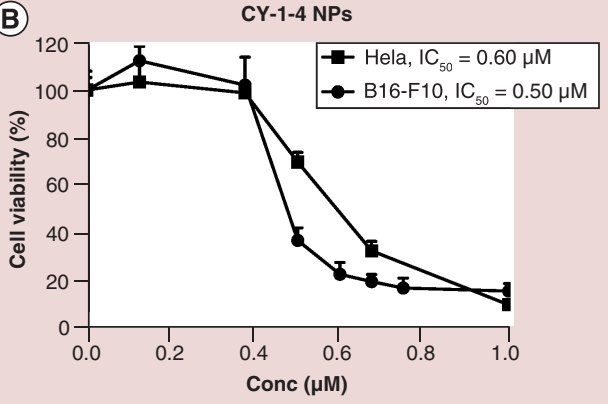

(C)
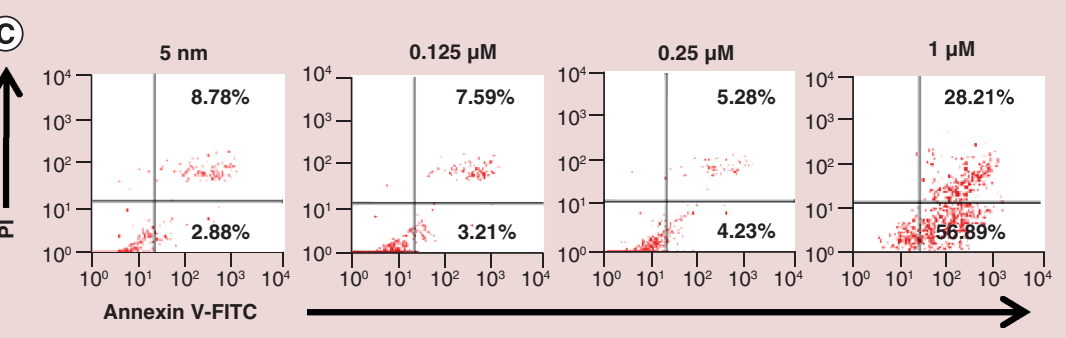

(D)
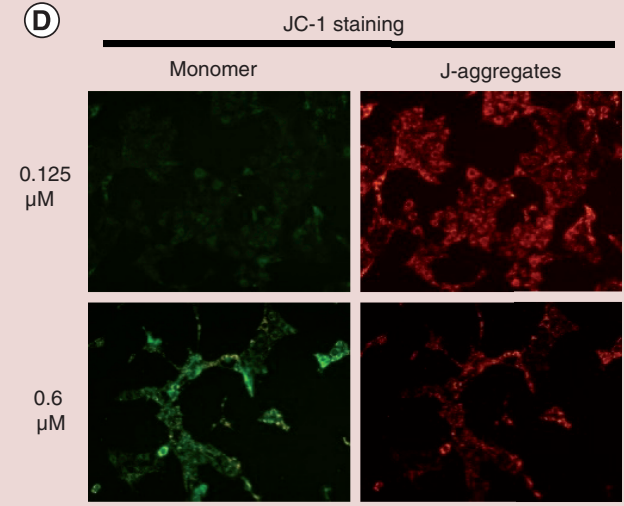

(E)
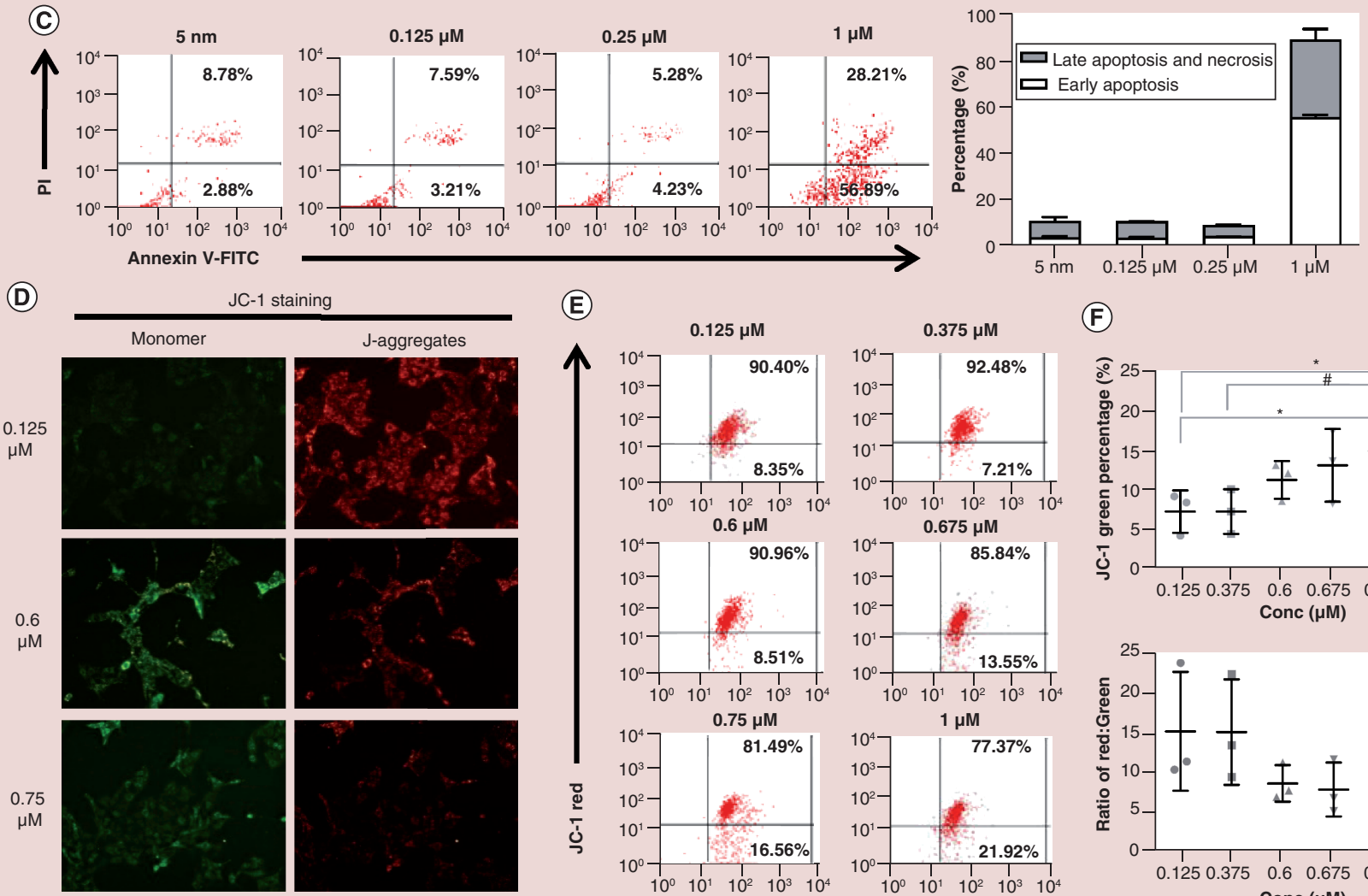

(G)
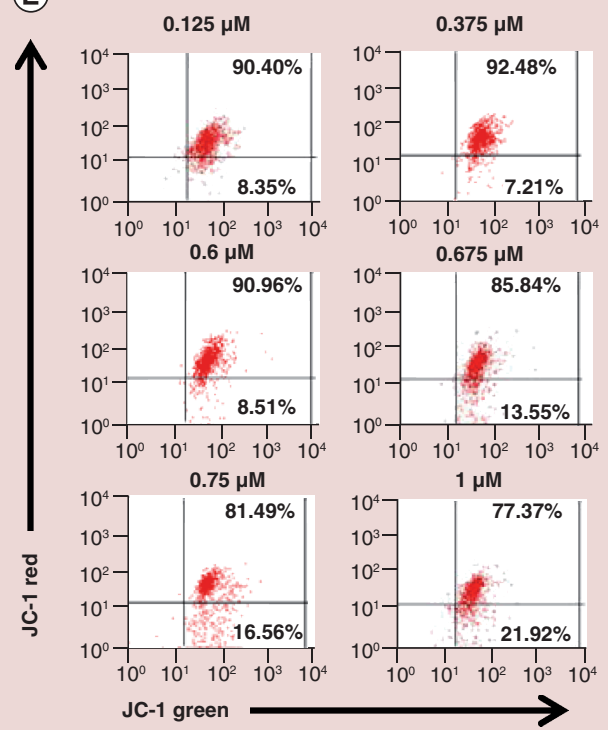

(F)
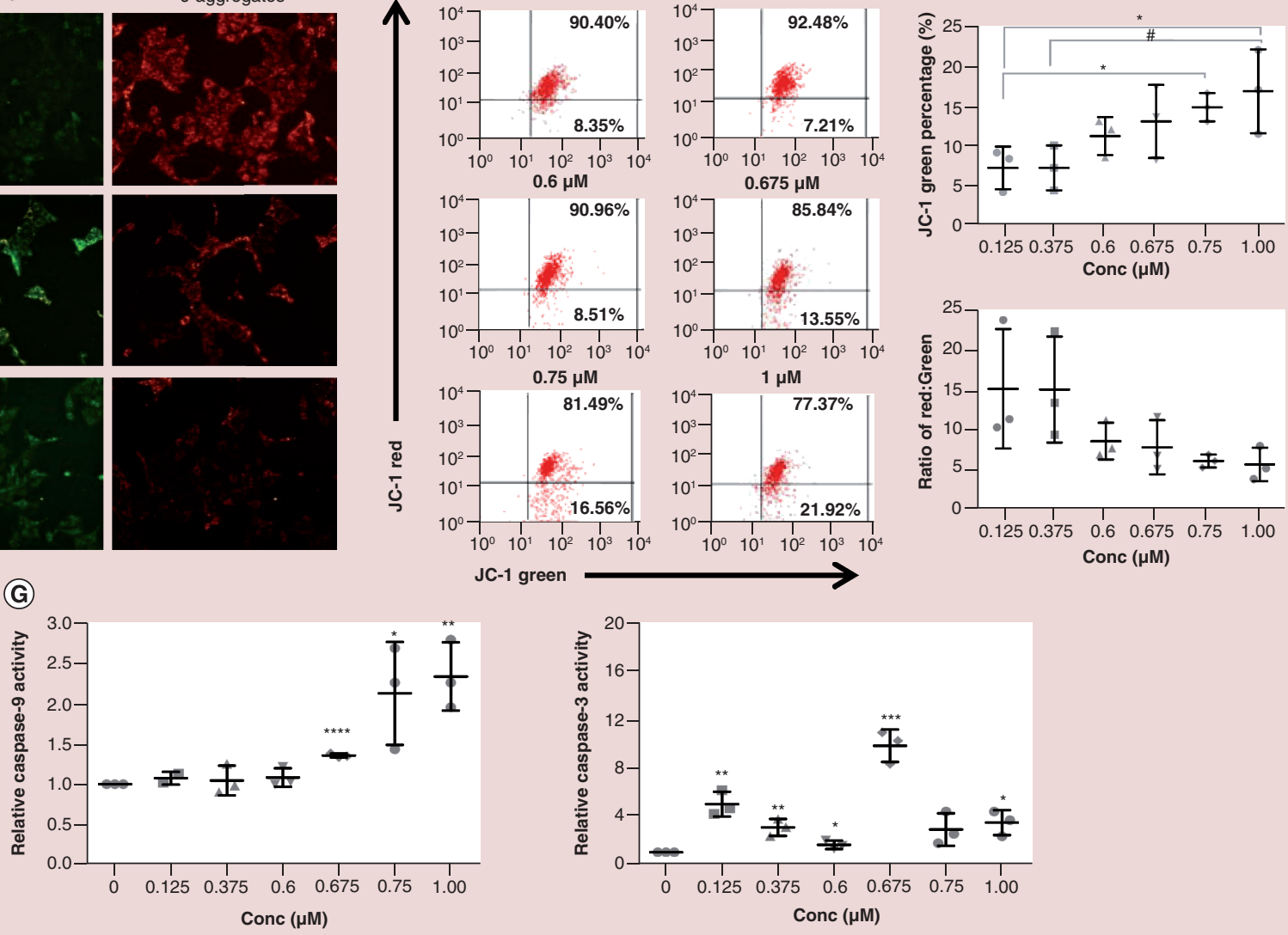

Figure 3. In vitro cytotoxicity of CY-1-4 free drug and CY-1-4 nanoparticles, and CY-1-4 nanoparticle induction of

mitochondrion-mediated cell death. (A) Cell viability associated with CY-1-4 free drug for HeLa, B16-F10 and LX-2 cells. (B) Cell viability associated with CY-1-4 NPs for HeLa cells and B16-F10 cells. IC 50 of each treatment for various cells is also presented. (C) (Left)

Representative dot plot of apoptosis by flow cytometry after the labeling with Annexin V-FITC and propidium iodide. (Right) Histogram for statistical analysis showed the quantitative ratio of early apoptosis and late apoptosis/necrosis upon treatment with $5 \mathrm{nM}, 0.125,0.25$ and $1 \mu \mathrm{M}$ CY-1-4 NPs. (D) Typical fluorescence photomicrographs of JC-1 staining output at concentrations of $0.125,0.6$ and $0.75 \mu \mathrm{M}$ CY-1-4 NPs; scale bars, $100 \mu \mathrm{m}$. (E) Representative dot plot of changed JC-1 fluorescence at various concentrations by flow cytometry. (F) Effect in percentage of JC-1 green and the ratio of JC-1 red over JC-1 green at different concentrations by flow cytometry. ${ }^{*} \mathrm{p}$

$<0.05$ versus $0.125 \mu \mathrm{M},{ }^{\#} \mathrm{p}<0.05$ versus $0.375 \mu \mathrm{M}$. (G) Relative caspase- 9 and caspase- 3 activity changes at different concentrations. ${ }^{*} \mathrm{p}<0.05 ;{ }^{* *} \mathrm{p}<0.01 ;{ }^{* * *} \mathrm{p}<0.001 ;{ }^{* * *} \mathrm{p}<0.0001$ versus $0 \mu \mathrm{M}$ as a control $(\mathrm{n}=3$, mean \pm standard deviation).

FITC: Fluorescein isothiocyanate; NP: Nanoparticle. 
got higher, especially at more than $0.675 \mu \mathrm{M}$. Activities of caspase-3 also further showed the downstream effect of apoptosis at various concentrations. These results cooperatively supported the idea that CY-1-4 NPs had the ability to induce cell death via a mitochondrion-mediated pathway.

\section{CY-1-4 NPs induce ICD}

To investigate the immunogenic effect of CY-1-4 NP-induced cell death, the damage-associated molecular patterns, including HMGB1, which were passively released from cell nuclei and CRT, which was exposed to the cell outer membrane, were tested using confocal laser scanning microscope and flow cytometry, respectively. Considering the cytotoxicity results, $0.75 \mu \mathrm{M}$ of CY-1-4 NPs was chosen as an appropriate cytotoxic concentration to test whether this induces ICD effectively. Compared with the control group, CY-1-4 NPs at $0.75 \mu \mathrm{M}$ induced significant extracellular release of HMGB1 from cell nuclei (Figure 4A), and the CRT-positive rate increased from 2.35 to $4.80 \%$ (Figure 4B). CY-1-4 free drug at the same concentration also moderately promoted HMGB1 release and increased the CRT-positive rate; however, in the NP group, stronger ICD was shown than that in the free drug group.

Furthermore, to examine the effect of immunogenic stimulation on DCs, we formed a coculture system of DC 2.4 cells and the culture medium of B16-F10 cells (including cell debris, after treatment with different concentrations of CY-1-4 NPs); DC 2.4 cells were successfully activated by them in a concentration-dependent manner. CD80 and CD86 are both costimulatory signal molecules highly expressed on mature DCs, which bind to $\mathrm{CD} 28$ on $\mathrm{T}$ cells to facilitate T-cell activation; CD11c is also a maturation marker of DC 2.4 cells. The rate of positivity for CD11c, CD80 and CD86 represented the activation of DCs. As Figure 4C \& D shows, CD11c was overexpressed in every treatment group, and the expression of CD86 was also dramatically increased as the concentration increased; especially when the concentration reached $0.675,0.75$ and $1 \mu \mathrm{M}$, the stimulation rates were boosted to near eightfold, tenfold and 20-fold in comparison with the untreated group. The expression of CD80 also changed significantly when the applied concentration reached 0.75 and $1 \mu \mathrm{M}$, and the expression of $\mathrm{CD} 80$ at $1 \mu \mathrm{M}$ was double that of the untreated group.

In addition, as Supplementary Figure 2 shows, after treatment with different concentrations of CY-1-4 NPs, the $\mathrm{IC}_{50}$ of DC 2.4 cells was $0.54 \mu \mathrm{M}$, which means that when the concentration was more than $0.375 \mu \mathrm{M}$, the cell viability was less than $50 \%$ and the cell phenotypes could not be estimated. Only the untreated group, the $0.125 \mu \mathrm{M}$ group and the $0.375 \mu \mathrm{M}$ group were tested for the surface phenotypes. However, the upregulation of $\mathrm{CD} 80^{+}$or $\mathrm{CD} 86^{+}$on DC 2.4 cells was rather low compared with that of DC 2.4 cells stimulated in the coculture system. These results indicate that the DC activation effect was not from CY-1-4 NP stimulation but from the dying tumor cell debris, which possessed ICD effects after treatment with CY-1-4 NPs.

\section{CY-1-4 NPs inhibited IDO at the enzymatic level \& cell-based assay}

IDO is the rate-limiting enzyme in the transformation from Trp to Kyn. First, we estimated IDO-inhibitory activity at the enzymatic level. The results showed that the $\mathrm{IC}_{50}$ value of CY-1-4 was $5.985 \mathrm{nM}$ (Figure $5 \mathrm{~A}$ ) ( $\mathrm{IC}_{50}$ of INCB024360 is $73 \mathrm{nM}$ and that of NLG919 is $38 \mathrm{nM}$ [25]), confirming its high IDO-inhibitory activity. Then, the activity of IDO was examined on tumor cells, by determining the generation of Kyn. To establish an IDO-overexpressing cell line, HeLa cells and B16-F10 cells were chosen to be stimulated by IFN- $\gamma$. The results in Figure 5B show that the absorbance in HeLa cells clearly increased after stimulation, indicating improved IDO activity. However, the B16-F10 cell line did not show any significant change in absorbance with or without IFN- $\gamma$, which means that these cells could not be successfully stimulated to generate more IDO. Similar results were further confirmed by directly determining the Kyn concentration in cultivation medium by HPLC (Supplementary Figure 3). Therefore, HeLa cells were chosen for in vitro IDO activity assay. The results in Figure 5C indicate the Kyn inhibition rate of CY-1-4 free drug and CY-1-4 NPs, which was an indicator of IDO activity inhibition. Both CY-1-4 free drug and CY-1-4 NPs showed moderate inhibitory efficacy at relatively low concentrations. As the concentration increased, a sharp increase $(0.375-0.5 \mu \mathrm{M}$ in the CY-1-4 free drug group and 0.5-0.75 $\mu \mathrm{M}$ in the CY-1-4 NP group) of Kyn inhibition was observed. The $\mathrm{IC}_{50}$ of CY-1-4 free drug was $0.30 \mu \mathrm{M}$ and it was $0.78 \mu \mathrm{M}$ for CY-1-4 NPs.

\section{CY-1-4 NPs generate better antitumor activity in therapeutic experiment}

To explore the antitumor efficacy and safety profile, CY-1-4 NPs and CY-1-4 free drug were set at three different doses (termed N1, N2, N3 and C1, C2, C3 for 150, 300 and $450 \mu \mathrm{g} / \mathrm{kg}$, respectively) for treatment in B16- 
(A)
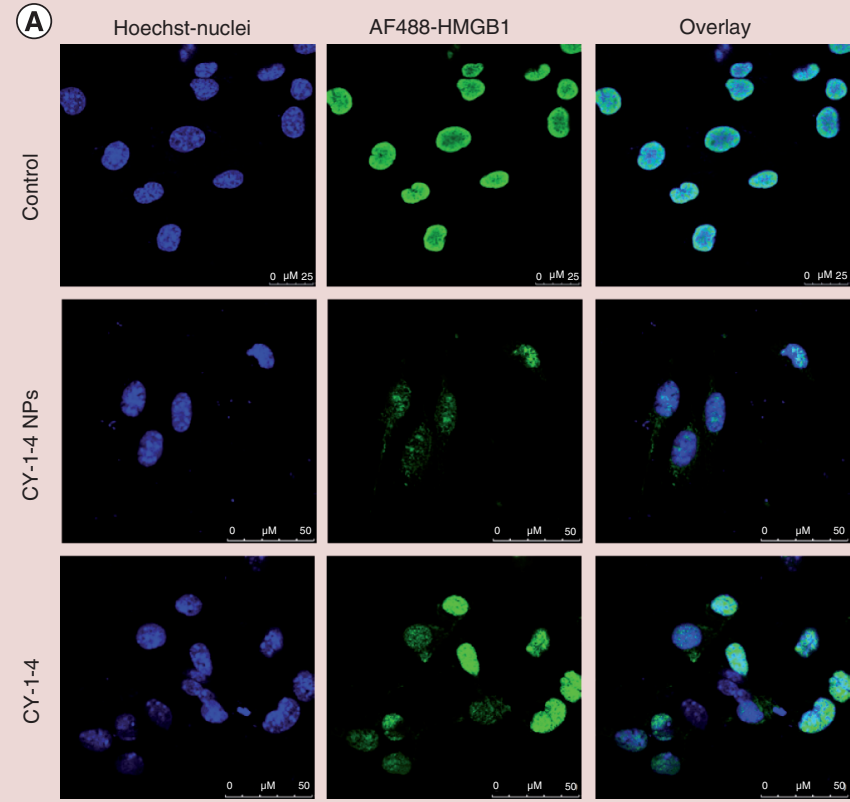

(B)

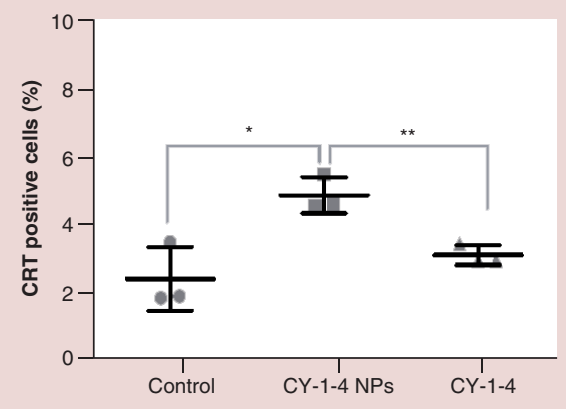

(C)
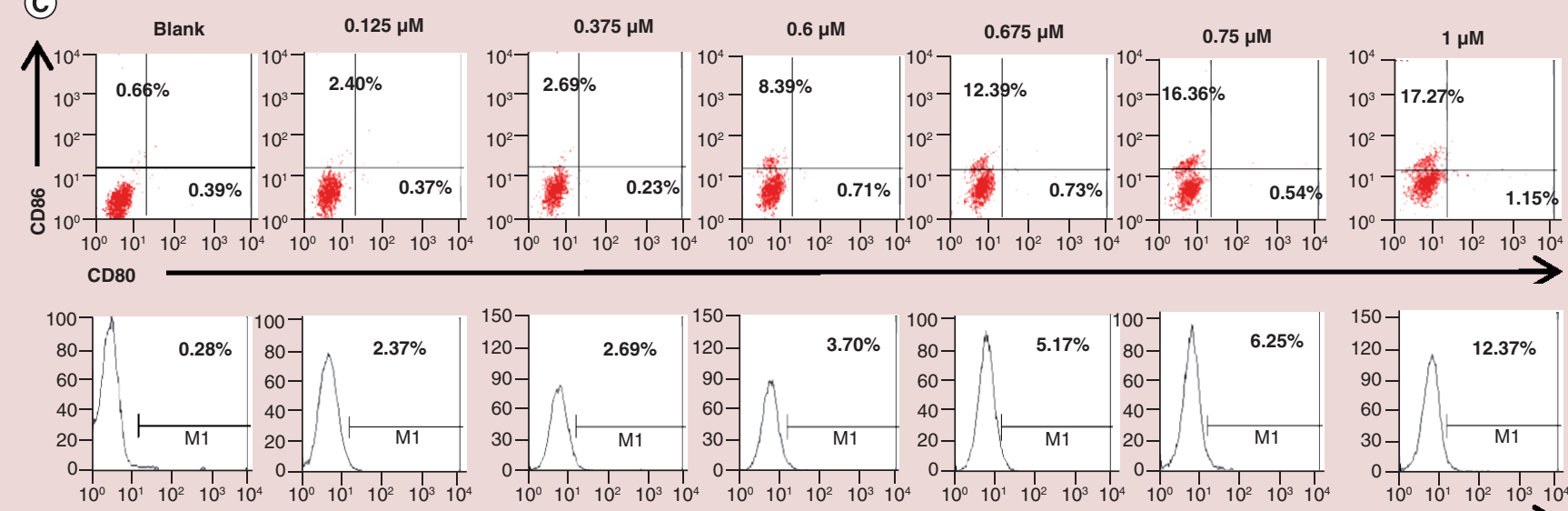

(D)
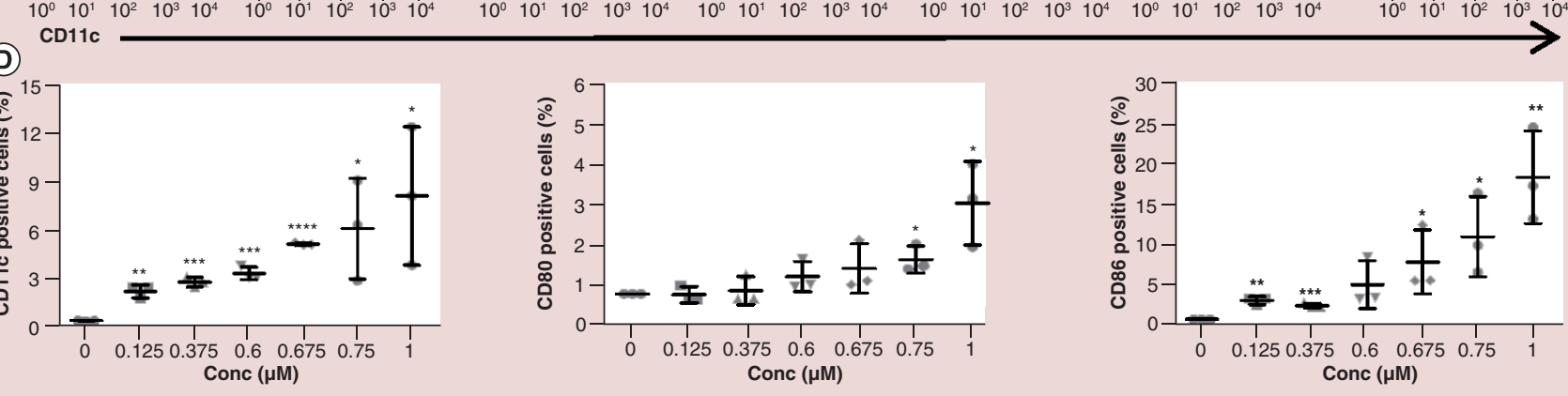

Figure 4. CY-1-4 nanoparticles induced immunogenic cell death. (A) Observation of the release of HMGB1 from the nucleus using confocal microscopy after treatment with $0.75 \mu \mathrm{M}$ CY-1-4 NPs or CY-1-4 free drug. Scale bar, $25 \mu \mathrm{m}$. (B) Percentage of CRT-positive cells quantified by flow cytometry after treatment with $0.75 \mu \mathrm{M}$ CY-1-4 NPs or CY-1-4 free drug. (C) Representative dot plot for the rates of positivity for CD11C, CD80 and CD86 by flow cytometry in DC 2.4 cells after treatment with different concentrations of CY-1-4 NPs in B16-F10 cells. (D) Statistical analysis of the rates of positivity for CD11C, CD80 and CD86 in each group above.

${ }^{*} \mathrm{p}<0.05 ;{ }^{*} \mathrm{p}<0.01 ; * * * \mathrm{p}<0.001 ; * * * * \mathrm{p}<0.0001$ versus $0 \mu \mathrm{M}$ as a control $(\mathrm{n}=3$, mean \pm SD).

CRT: Calreticulin; NP: Nanoparticle. 
(A)

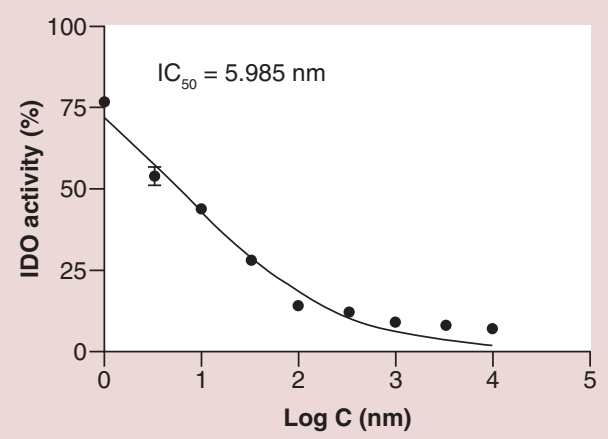

(B)

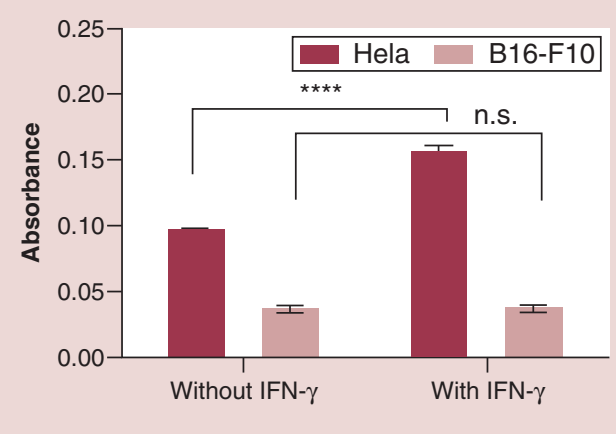

(c)

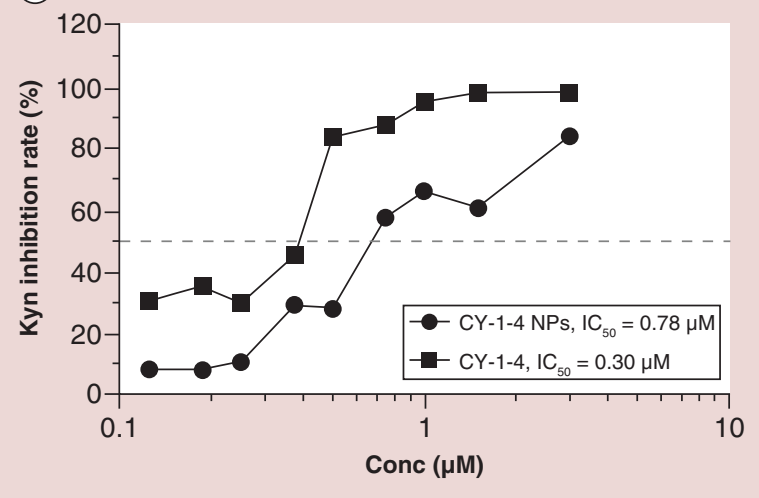

Figure 5. The enzymatic IDO-inhibitory activity of CY-1-4, and the IDO-inhibitory efficacy of treatment with CY-1-4 free drug and CY-1-4 nanoparticles in HeLa cells. (A) Effect on IDO activity of CY-1-4 at the enzymatic level. (B) Absorbance at $490 \mathrm{~nm}$ of kynurenine generated in HeLa cells and B16-F10 cells when treated with or without IFN- $\gamma$ by colorimetric assay. (C) Cell-based effect on kynurenine production inhibition rate of CY-1-4 free drug and CY-1-4 NPs ( $n=3$, mean \pm standard deviation).

NP: Nanoparticle.

F10 cell-bearing C57BL6 mice. As shown in Figure 6A \& Supplementary Figure 4, CY-1-4 free drug at all concentrations did not elicit significant tumor inhibition ability compared with the PBS-treated group. However, Figure 6B \& Supplementary Figure 4 indicate that the N2 group of CY-1-4 NPs at $300 \mu \mathrm{g} / \mathrm{kg}$ generated optimal inhibition of tumor growth, and the N1 group showed moderate inhibition capability. Notably, in the group with a higher concentration of CY-1-4 NPs $(450 \mu \mathrm{g} / \mathrm{kg})$, negligible antitumor activity was exhibited, with the tumor volume being almost the same as in the PBS group. These results clarified that the medium concentration of CY-1-4 NPs elicited the best tumor-inhibiting efficacy. H\&E staining of heart, liver, spleen, lung and kidney showed that, in the N3 group, slight edema appeared in the liver and spleen, while in the other groups, no significant damage was elicited and good safety was observed (Supplementary Figure 5).

IDO expression and apoptosis in tumors were further evaluated. The corresponding representative IHC images are presented in Figure 6C \& E, respectively. Each immunostained section was fully scanned (see Supplementary Figures $6 \& 7$ ) and then three views were randomly selected at a magnification of $20 \times$ to quantitatively examine the positivity of IDO and TUNEL. As shown in Figure 6D, CY-1-4 free drug at low and medium doses (C1, C2) showed little reduction of IDO positivity, while CY-1-4 free drug at a high dose (C3) elicited a moderate decrease in IDO expression. Moreover, a significant decrease of IDO expression could be seen in CY-1-4 NPs at low and medium doses $(\mathrm{N} 1, \mathrm{~N} 2)$, especially in the N2 group. However, in the N3 group, there was a less apparent decrease in IDO expression level. According to the TUNEL analysis, all groups showed some region of TUNEL-positive cells (Figure 6E). Among these, CY-1-4 free drug at a high dose (C3) showed the highest positivity for apoptosis, which was fourfold compared with that in the PBS group. Moreover, CY-1-4 NPs at a high dose also caused an 


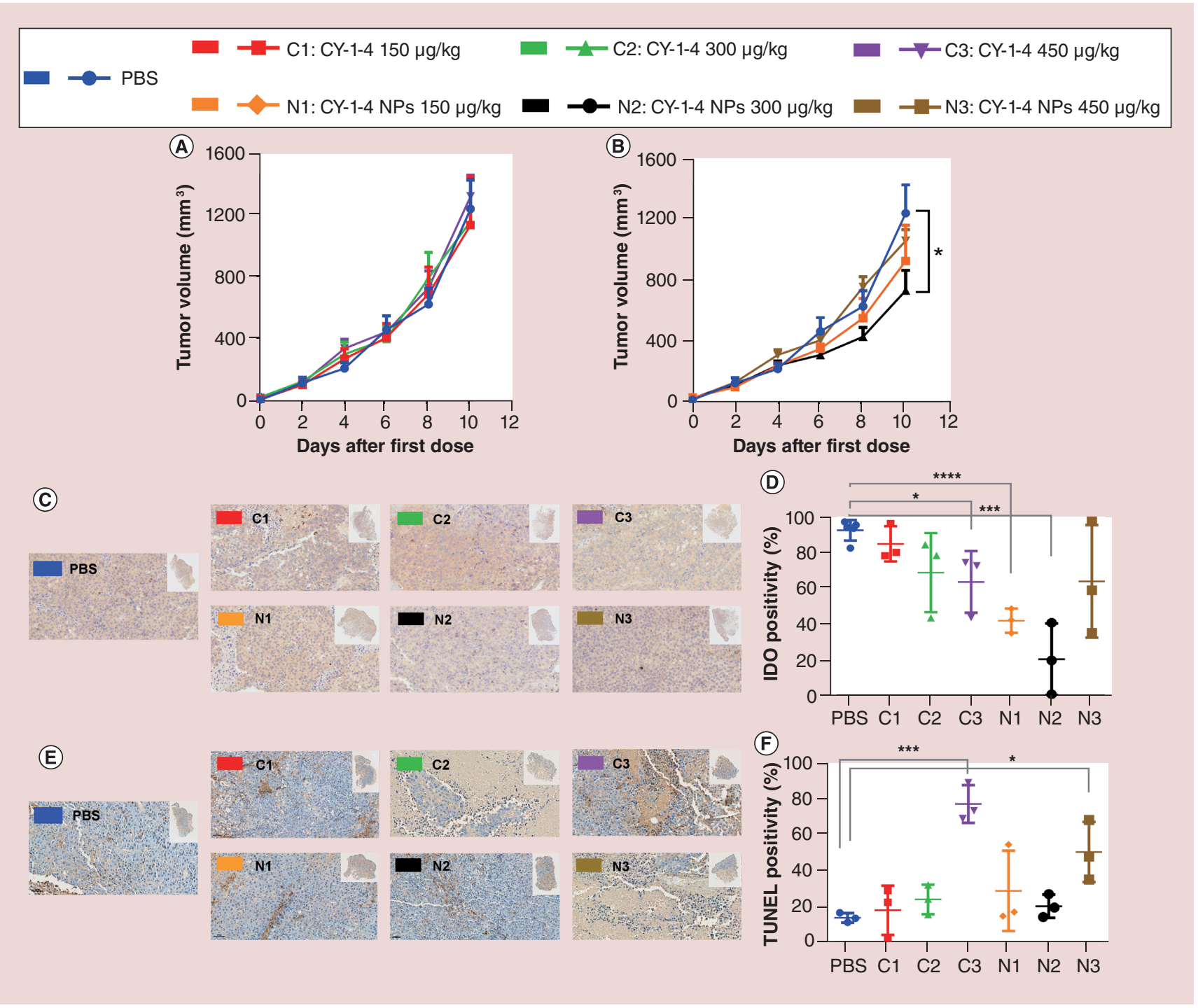

Figure 6. Effects of CY-1-4 free drug and CY-1-4 nanoparticles on tumor tissue growth inhibition, IDO expression and tumor cell apoptosis. Tumor tissue growth after treatment with (A) CY-1-4 free drug and (B) CY-1-4 NPs at various doses in the B16-F10 tumor model. ${ }^{*} p<0.05$ versus PBS group ( $n=7$, mean \pm standard error of mean). (C) Representative immunohistochemical graphs for IDO staining and (D) quantitative analysis of IDO positivity (\%) in different treatment groups. Full scan graphs are presented in the top right corner. (E) Representative immunohistochemistry graphs for TUNEL staining and (F) quantitative analysis of TUNEL positivity (\%) in different treatment groups. Full scan graphs are presented in the top right corner. Scale bar, $50 \mu \mathrm{m}$. ${ }^{*} \mathrm{p}<0.05,{ }^{* * *} \mathrm{p}<0.001, * * * * p<0.0001$ versus PBS group ( $n=3$, mean \pm standard deviation).

NP: Nanoparticle; PBS: Phosphate-buffered saline; TUNEL: Terminal deoxynucleotide transferase-mediated dUTP-biotin nick-end labelling.

extensive number of apoptosis-positive cells. These two high doses both confirmed the cytotoxicity of CY-1-4 at a high dose, irrespective of the form used. In addition, CY-1-4 at low and medium doses in the forms of free drug and NPs did not elicit apparent apoptosis in comparison with the PBS group, which means that they did not induce strong cell death at such doses (Figure 6F).

CY-1-4 NPs at medium concentration elicit dual tumor inhibition activity

To further investigate the IDO inhibitory activity of CY-1-4, the in vivo antitumor efficacy of CY-1-4 NPs at a dose of $300 \mu \mathrm{g} / \mathrm{kg}$ was compared with that of 1-MT. Compared with the PBS and 1-MT groups, CY-1-4 NPs clearly inhibited the tumor growth (Figure $7 \mathrm{~A}-\mathrm{C}$ ). At the end point, the inhibition of tumor size was about $42 \%$ compared with that in the PBS group. 1-MT barely inhibited tumor growth compared with that in the PBS group, which 


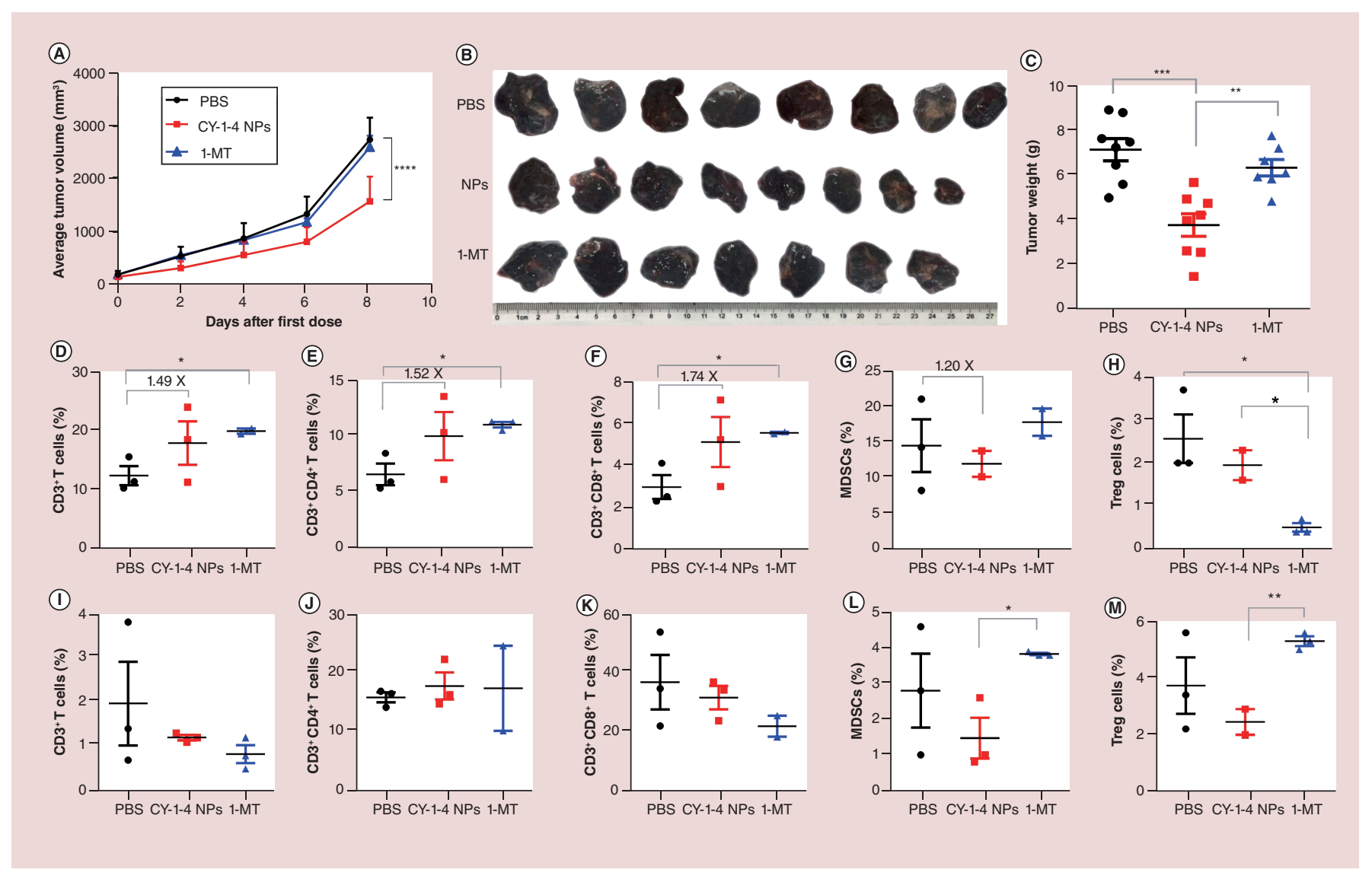

Figure 7. Effect of CY-1-4 nanoparticles on tumor growth inhibition and immune cell subset changes. (A) Tumor growth kinetics and (B) photographs from tumor-bearing mice in each group. (C) Average tumor weight of tumor-bearing mice in different treatment groups. ${ }^{* *} \mathrm{p}<0.01, * * * \mathrm{p}<0.001, * * * * \mathrm{p}<0.0001$ ( $\mathrm{n}=8$, mean \pm standard error of mean). (D-H) Flow cytometry analysis of immune cell subsets in spleen. Percentages of (D) $\mathrm{CD}^{+} \mathrm{T}$ cells, (E) CD4 ${ }^{+} \mathrm{T}$ cells, (F) CD8 ${ }^{+} \mathrm{T}$ cells, (G) $\mathrm{CD} 11 \mathrm{~b}^{+} / \mathrm{Gr}-1^{+}$myeloid-derived suppressive cells and (H) $\mathrm{CD} 4^{+} \mathrm{CD} 25^{+} \mathrm{FoxP}^{+}$Treg cells of each group were determined. (I-M) Flow cytometry analysis of immune cell subsets in tumor tissues. Percentages of (I) CD3 ${ }^{+} \mathrm{T}$ cells, (J) CD4 ${ }^{+} \mathrm{T}$ cells, (K) CD8 ${ }^{+} \mathrm{T}$ cells, (L) CD11 b $/ \mathrm{Gr}-1^{+}$myeloid-derived suppressive cells and (M) $\mathrm{CD} 4{ }^{+} \mathrm{CD} 25^{+} \mathrm{FoxP}^{+}$Treg cells of each group were detected. ${ }^{*} \mathrm{p}<0.05,{ }^{*} \mathrm{p}<0.01$ ( $\mathrm{n}=3$, mean \pm standard error of mean). N2: CY-1-4 nanoparticle; NP: Nanoparticle; PBS: Phosphate-buffered saline.

was similar to that in previous research [9]. None of the treatments caused a decrease of bodyweight, illustrating their safety.

To clarify the effects of CY-1-4 NPs on the tumor immune microenvironment, the immune cell subpopulations in spleens and tumors were analyzed by flow cytometry 1 day after completing the last dose administration. Compared with the levels in tumor-free mice, $\mathrm{CD} 3^{+} \mathrm{T}$ cells, $\mathrm{CD} 4^{+} \mathrm{T}$ cells and $\mathrm{CD} 8^{+} \mathrm{T}$ cells were significantly reduced in tumor-bearing mice, and inhibitory immune cells, such as Tregs and MDSCs, both infiltrated more into the spleens of tumor-bearing mice (Supplementary Figure 8). These results indicate that tumorigenesis indeed destroyed the immune homeostasis. Figure 7D-H and Supplementary Figure 9 show the results of immune cell subpopulations in spleens with different treatments in tumor-bearing mice. It was noted that, after treatment with CY-1-4 NPs or 1-MT, more infiltration of $\mathrm{CD}^{+} \mathrm{T}$ cells, $\mathrm{CD}^{+} \mathrm{T}$ cells and $\mathrm{CD}^{+} \mathrm{T}$ cells occurred in mouse spleens compared with the level in the control group (Figure 7D-F). In addition, CY-1-4 NPs slightly reduced the recruitment of MDSCs compared with those in the PBS group and 1-MT group (Figure 7G). Finally, Tregs associated with CY-1-4 NPs were also moderately decreased in comparison with those in the PBS group. Meanwhile, 1-MT showed better inhibitory efficacy on Tregs (Figure 7H). These results indicate that CY-1-4 NPs had regulatory activity on antitumor immunosuppression throughout the body.

Figure 7I-M and Supplementary Figure 10 show the results of flow cytometry analysis of immune cell subpopulations in tumor tissues. It was noted that the percentage of total T cells decreased in both the CY-1-4 NP and 1-MT groups; these decreases may have resulted from the cytotoxicity of drugs. However, $\mathrm{CD} 4^{+} \mathrm{T}$ and $\mathrm{CD} 8^{+} \mathrm{T}$ cells 
were at almost the same level in CY-1-4 NPs compared with the level in the PBS group, which meant that the ratio of $\mathrm{CD}^{+} \mathrm{T}$ and $\mathrm{CD} 8{ }^{+} \mathrm{T}$ cells was relatively increased in the CY-1-4 NP group; this could have been the effect of inhibiting IDO and cytotoxic T-cell production (Figure 7I-K). Moreover, the CY-1-4 NP group showed much less MDSC recruitment in the tumor tissues (Figure 7L) compared with the levels in the PBS and 1-MT groups. This is consistent with previous research showing that the inhibition of IDO leads to reduced MDSCs in tumors [26]. Treg infiltration in tumor tissues was also cut upon treatment with CY-1-4 NPs (Figure 7M), finally establishing a refined tumor immuno-microenvironment with increased cytotoxic $\mathrm{T}$ lymphocytes and fewer inhibitory immune cells. However, Tregs and MDSCs remained unchanged upon treatment with 1-MT. In conclusion, these results show that CY-1-4 NPs effectively reduced tumor size in dual functions of chemotherapy and immunotherapy.

\section{Discussion}

Inspired by the fact that antimalarial parasite drugs such as chloroquine and artemisinin have antitumor activity through a mitochondrion-dependent pathway via heme $[27,28]$ and that heme plays a crucial role in the effects of IDO inhibitors such as NLG919 and INCB024360 [29-31], we hypothesized that the antimalarial parasite drug candidate tryptanthrin derivative CY-1-4 (Figure 2B) has both antitumor activity and IDO-inhibitory activity. The antitumor activity of tryptanthrin has been reported previously $[32,33]$ and tryptanthrin derivatives were also recently reported to inhibit IDO with therapeutic activity in Lewis lung cancer tumor-bearing mice [4]. However, tryptanthrin and its derivatives have not been extensively taken into consideration for pharmaceutical development because of their poor solubility [34]. Therefore, we first developed an effective NP-based delivery system to encapsulate and deliver CY-1-4. PCL as an FDA-approved biomedical polymer carried CY-1-4 through hydrophobic interaction and improved its solubility; additional 10\% PEG-modified PCL could protect CY-1-4 NPs from opsonization and reticuloendothelial system clearance [35], thus prolonging the circulation time to reach tumor sites in the body. The appropriate size of the CY-1-4 NPs also helped them passively target tumor tissue because of the EPR effect [36].

In cytotoxicity study, both free drug and NPs showed lower $\mathrm{IC}_{50}$ in tumor cells (HeLa and B16-F10 cells) and higher IC $_{50}$ in normal cells (LX-2), indicating the tumor selectivity of CY-1-4 (Figure 3A \& B). Further mechanismrelated experiments suggested that CY-1-4 at a higher concentration caused MMP change and increases in caspase-9 and caspase-3 activity, inducing cell death in a mitochondrion-dependent pathway (Figure 3C-G). These results are consistent with a study that reported that tryptanthrin could cause marked swelling, mitochondrial destruction and apoptosis in U-937 and HL-60 cells [5]. Interestingly, we further found that CY-1-4 could induce ICD and NP encapsulation would potentiate the ICD effect. CY-1-4 free drug at $0.75 \mu \mathrm{M}$ showed modest damage-associated molecular patterns (HMGB1 exposure and CRT-positive rate), while NPs not only offered a good opportunity for efficient delivery of CY-1-4 to tumor tissues but also acted as an 'adjuvant,' helping CY-1-4 free drug induce more HMGB1 release and CRT exposure compared with free drug at the same concentration. This phenomenon was supported by research showing that mPEG-PLGA NPs would enhance the ICD effect of oxaliplatin [10]. Moreover, the tumor cell debris treated with high concentrations of CY-1-4 NPs could activate DCs to express CD80 and CD86, suggesting a stronger immune stimulatory effect (Figure 4). To our knowledge, this is the first report of a tryptanthrin derivative-mediated effect on ICD.

In the IDO inhibition study, CY-1-4 showed strong ability to inhibit IDO activity at the enzymatic level on the nanomole order, which was much higher than for INCB024360 and NLG919 [25], despite them having similar heme-combining inhibitory mechanisms [29-31]. In cell-based assay, CY-1-4 free drug and CY-1-4 NPs both inhibited kynurenine production in a concentration-dependent manner, which was an indicator of IDO activity inhibition (Figure 5). These results confirmed that the tryptanthrin derivatives have IDO-inhibitory activity. Moreover, inspired by CY-1-4, we speculated on a new research direction that the existing antimalarial parasite drugs that act by inhibiting heme polymerization may also possess IDO-inhibitory activity. This observation may narrow the scope for screening potential IDO inhibitors and decrease the limit of druggability, finally achieving 'old drug new use.'

Experiments in vivo explored the antitumor ability of CY-1-4 free drug and CY-1-4 NPs at three different doses on B16-F10 tumor-bearing mice, revealing some interesting phenomena: First, CY-1-4 free drug at all doses did not show any tumor growth inhibition ability, although it did show good cytotoxicity and IDO-inhibitory ability in vitro (Figure 6A). This might be because simple solubilization of CY-1-4 free drug in DMSO may cause rapid clearance due to its short half-life; alternatively, the dramatically changed liquid medium in vivo might cause crystallization of the free drug, which could be cleaned by reticuloendothelial system and could not accumulate sufficiently in tumor tissues. Moreover, crystallization might also result in heterogeneity of permeation even if the 
free drug at a higher dose (C3) partly reached tumor tissues. As for CY-1-4 NPs, they all effectively inhibited tumor growth, although the doses were only 150,300 and $450 \mu \mathrm{g} / \mathrm{kg}$. Generally, to achieve antitumor efficiency, the dose of oral administration of tryptanthrin derivatives reached nearly $200 \mathrm{mg} / \mathrm{kg}$ [4]. This result further proved the reasonability of our drug delivery system design. That is to say, the nanodrug delivery system CY-1-4 NPs could prolong the circulation time of CY-1-4 and promote CY-1-4 passively targeting tumor tissues via the EPR effect, and NPs provided a dose gradient of CY-1-4 in different parts inside tumors, thus showing better comprehensive antitumor efficacy. However, in the CY-1-4 NP groups, it was notable that the medium-dose group (N2) showed maximal inhibition, but the higher-dose group did not (N3), although they did not show a significant difference ( $\mathrm{p}$ $=0.063$; Figure 6B). It was easy to understand why the low-dose group (N1) showed the least antitumor efficiency because a low dose might only inhibit IDO, and the single effect was not strong enough to combat the complicated and suppressive tumor microenvironment. However, given the obtained findings, the following question arises: why did the best tumor reduction efficacy not occur in the high-dose group (N3)?

We thought that this might be related to the status of the immune system. The immune system plays an essential role in antitumor chemotherapy and drug dose can have a large effect on immunomodulatory activity [37]. First, as heme-blocking compounds, the targeting by CY-1-4 NPs of heme occurred not only in tumor cells but also in neighboring immune cells, such as tumor-associated macrophages (with an M2 phenotype). M2-like macrophages mainly act in scavenging extracellular heme and digesting intracellular heme to release free ferrous iron for recycling, mediating an anti-inflammatory immune response $[38,39]$. Therefore, we speculated that targeting intracellular heme in M2 macrophages by a lower amount of CY-1-4 might reduce the free ferrous iron release, slowing the growth rate of neighboring tumor cells, which require more iron than other cells. Second, iron accumulation in M2 macrophages contributes to the induction of the proinflammatory M1 phenotype [38], which may help in the fight against tumor cells. However, at the higher dose of CY-1-4 NPs, too much heme accumulation intracellularly might cause high toxicity of macrophages [40], and possibly high cytotoxicity might cause lymphodepletion [41] such as of lymphocytes or myeloid cells, impairing the endogenous antitumor response, which might act against the remaining cancer cells.

Immunohistochemical results of IDO expression and TUNEL assay in tumor tissues further supported the above results. CY-1-4 free drug did not significantly reduce IDO positivity in tumor tissues, and the apparent decrease of IDO positivity in N1 and N2 groups suggested that NPs effectively carrying CY-1-4 accumulated in tumor tissues and reduced local IDO expression, while N3 did not reduce IDO expression as much as in the N2 group (Figure 6D). It was previously reported [4] that tryptanthrin derivatives could inhibit IDO activity but increase IDO expression because a compensatory counter-regulatory mechanism was activated by IDO inhibitors. However, it was also reported that some IDO inhibitors (such as INCB024360) completely blocked IDO enzymatic activity but did not change IDO expression in LPS- or IFN- $\gamma$-treated DCs [18]. In our study, we first observed the doseeffect interaction between drug dose and IDO expression. We speculate that too high a dose may increase IDO expression because of cytotoxicity in tumor immune cells, but when applied at an appropriate lower dose (N2), IDO expression could decrease. Moreover, TUNEL positivity confirmed the dose-dependent cell death effect, and C3 and N3 groups showed dramatically high apoptosis rates (Figure 6F).

Notably, in many researches which related to immunotherapy, most tumor-bearing mouse models are melanoma models. However, in our study, it was found that B16-F10 cells could not be stimulated to produce more kynurenine when stimulated by IFN- $\gamma$ in a cell-based assay. However, IDO could be detected to be overexpressed in melanoma tumor tissues. These inconsistencies between in vitro and in vivo may indicate that IDO was not always expressed and could not be stimulated by IFN- $\gamma$ in all kinds of tumor cell lines, and the sensitivity of cell lines in IDO stimulation was also not the same. In tumor tissues, the expression of IDO in tumor cells and other neighboring immune cells was a result of the complicated tumor microenvironment and plasmacytoid dendritic cells appeared to be responsible for host IDO expression [42].

Immune cell subsets in spleen and in tumor tissues illustrated the immune microenvironment in mice. Although 1-MT is an IDO signal pathway inhibitor and not a valid inhibitor of IDO1 enzyme activity, it is a small-molecule drug that is now the subject of the most intense study in IDO inhibition, so we chose it as a reference of similar antitumor efficacy. First, the promotion of Th cells $\left(\mathrm{CD}^{+} \mathrm{T}\right.$ cells) and effector $\mathrm{T}$ cells $\left(\mathrm{CD} 8^{+} \mathrm{T}\right.$ cells) by CY-1-4 NPs and 1-MT (Figure 7E \& F) in spleen indicated that IDO inhibition could establish a stronger systematic immune environment to fight against cancer in the body. The significant reduction of Tregs in the spleen of the 1-MT group illustrated that this classical IDO inhibitor had good regulatory function in a suppressive microenvironment. However, the inefficiency of 1-MT in reducing tumor size in our experiment (Figure 7C) was 
consistent with a previous report [9]. Further immunological analysis in tumor tissues (Figure 7L \& M) indicated that the 1-MT group could not reduce the suppressive MDSC recruitment in tumor and hardly decrease Treg quantity locally, which meant that the immunological regulatory efficacy did not occur in local tumor tissues due to the lack of targeting ability. In contrast, CY-1-4 NPs simultaneously showed immunological effects in tumor tissues. The reduction in the percentage of total intratumoral T cells in the CY-1-4 NP group relative to the PBS group might have been the heme-blocking effect or cytotoxicity on these immune cells. However, the higher ratio of CD4 ${ }^{+} \mathrm{T}$ cells and $\mathrm{CD} 88^{+} \mathrm{T}$ cells in the CY-1-4 NP group further demonstrated enhanced infiltration of effector $\mathrm{T}$ cells in tumor tissues. Moreover, the significantly reduced MDSC recruitment and Treg quantity compared with those in the 1-MT group supported the effectiveness of local inhibition in tumor sites, and these reductions may also relate to the cytotoxic effect in immune cells [37]. Taking all of these findings together, CY-1-4 NPs at a medium dose achieved the initially designed advantage of an NP delivery system: passive targeting by the EPR effect to potentiate local antitumor efficacy. Consequently, CY-1-4 NPs established a stronger systematic and local immune microenvironment and effectively potentiated antitumor efficacy.

\section{Conclusion}

In this study, an effective drug delivery system to carry tryptanthrin derivative CY-1-4 to tumor tissues was developed, for improving antitumor efficacy with dual functions of immunotherapy and chemotherapy. Our study raises the possibility that antimalarial parasite drugs targeting heme could have IDO-inhibitory ability, providing a better perspective and reference to screening antitumor candidates; we also concluded that different doses of CY-1-4 NPs would influence IDO expression in the opposite direction. The use of CY-1-4 NPs involves the combination of immunotherapy and chemotherapy in a single therapeutic agent and offers a nanomedicine approach to achieve antitumor efficacy. It also prompts us to think about the balance between cytotoxicity and the special activity of compounds in certain applications.

\section{Future perspective}

The discovery and rational exploitation of IDO inhibitors should become a hot topic. Inspired by CY-1-4 NPs, the IDO-inhibitory potential of other antimalarial parasite drugs that possess heme-related mechanisms of action warrants further study. Moreover, a heme-blocking effect on the microenvironment may be a potential target to treat cancers.

\section{Summary points}

- IDO has become a crucial target for antitumor immunotherapy and some tryptanthrin derivatives have been reported to have IDO-inhibitory activity.

- CY-1-4 is a tryptanthrin derivative. Nanoparticle CY-1-4 improved the solubility of CY-1-4, achieving enhanced permeability and retention effect in tumors.

- Nanoparticle CY-1-4 could kill cancer cells by mitochondrion-mediated cytotoxicity and heme-blocking effect on both tumor cells and immune cells and could inhibit IDO activity in tumor and induce immunogenic cell death (ICD) to activate immature dendritic cells.

- Nanoparticle CY-1-4 combines immunotherapy and chemotherapy into a single therapeutic agent and offers a nanomedicine approach to achieve antitumor efficacy.

Supplementary data

To view the supplementary data that accompany this paper please visit the journal website at: www.futuremedicine.com/doi/full/10.2217/nnm-2019-0074

Author contributions

Authors' contributions are in the following order: conception and design: C Yang, X Wang, X Meng, B He; development of methodology: C Yang, Q Zheng; acquisition of data (provided animals, acquired and managed patients, provided facilities, etc.): $C$ Yang, Q Zheng, D Wang, M Qin; analysis and interpretation of data (e.g., statistical analysis, biostatistics, computational analysis): C Yang, Q Zheng, X Wang, B He; writing, review, and/or revision of the manuscript: C Yang, X Wang, B He, X Meng; administrative, technical or material support (i.e., reporting or organizing data, constructing databases): X Wang, X Meng, Q Zhang, B He, H Zhang, W Dai and study supervision: X Wang, X Meng. 
Financial \& competing interests disclosure

This work was supported by the National Natural Science Foundation of China (grant numbers: 31671017, 81872809), the National Key Research and Development Program of China (grant number: 2017YFA0205600) and Beijing Natural Science Foundation (grant number: 7162108). The authors have no other relevant affiliations or financial involvement with any organization or entity with a financial interest in or financial conflict with the subject matter or materials discussed in the manuscript apart from those disclosed.

Writing support was provided by Enago and was funded by the above funding sources.

\section{Ethical conduct of research}

All animal experiments were carried out according to protocols approved by the Committee for Animal Research of Peking University, China. The operations were conformed to the Guide for the Care and Use of Laboratory Animals (NIH publication no. 86-23, revised 1985). The authors state that they have obtained appropriate institutional review board approval or have followed the principles outlined in the Declaration of Helsinki for all human or animal experimental investigations.

\section{Open access}

This work is licensed under the Attribution-NonCommercial-NoDerivatives 4.0 Unported License. To view a copy of this license, visit http://creativecommons.org/licenses/by-nc-nd/4.0/

\section{References}

Papers of special note have been highlighted as: $\bullet$ of interest; $\bullet \bullet$ of considerable interest

1. Uyttenhove C, Pilotte L, Théate I et al. Evidence for a tumoral immune resistance mechanism based on tryptophan degradation by indoleamine 2,3-dioxygenase. Nat. Med. 9(10), 1269-1274 (2003).

2. Munn DH, Mellor AL. IDO in the tumor microenvironment: inflammation, counter-regulation, and tolerance. Trends Immunol. 37(3), 193-207 (2016).

3. Peng Y, Ueng S, Tseng C et al. Important hydrogen bond networks in indoleamine 2,3-dioxygenase 1 (IDO1) inhibitor design revealed by crystal structures of imidazoleisoindole derivatives with IDO1. J. Med. Chem. 59(1), 282-293 (2016).

4. Yang S, Li X, Hu F et al. Discovery of tryptanthrin derivatives as potent inhibitors of indoleamine 2,3-dioxygenase with therapeutic activity in Lewis lung cancer (LLC) tumor-bearing mice. J. Med. Chem. 56(21), 8321-8331 (2013).

- Presented some similar tryptanthrin deriviates with indoleamine 2,3-dioxygenase (IDO) inhibition ability.

5. Kimoto T, Hino K, Koya-Miyata S et al. Cell differentiation and apoptosis of monocytic and promyelocytic leukemia cells (U-937 and HL-60) by tryptanthrin, an active ingredient of Polygonum tinctorium Lour. Pathol. Int. 51(5), 315-325 (2010).

6. Pathania AS, Suresh K, Guru SK et al. The synthetic tryptanthrin analogue suppresses STAT3 signaling and induces caspase dependent apoptosis via ERK up regulation in human leukemia HL-60 cells. Eur. J. Cancer 50(11), S141-S141 (2014).

7. Hicks R, Nichols DA, Ditusa C et al. Evaluation of 4-azaindolo[2,1- b ]quinazoline-6,12-diones' interaction with hemin and hemozoin: a spectroscopic, x-ray crystallographic and molecular modeling study. Internet Electron. J. Mol. Des. 4, 751-764 (2005).

-. Provides a detailed hem-binding mechanisms of CY-1-4.

8. Brochez L, Chevolet I, Kruse V. The rationale of indoleamine 2,3-dioxygenase inhibition for cancer therapy. Eur. J. Cancer 76, 167-182 (2017).

\section{- Describes combination therapy.}

9. Hou D, Muller AJ, Sharma MD et al. Inhibition of indoleamine 2,3-dioxygenase in dendritic cells by stereoisomers of 1-methyl-tryptophan correlates with antitumor responses. Cancer Res. 67(2), 792-801 (2007).

\section{- Described the nanoparticle strategy to enhance the immunogenic cell death.}

10. Zhao X, Yang K, Zhao R et al. Inducing enhanced immunogenic cell death with nanocarrier-based drug delivery systems for pancreatic cancer therapy. Biomaterials 102, 187-197 (2016).

11. Maeda H, Wu J, Sawa T et al. Tumor vascular permeability and the EPR effect in macromolecular therapeutics: a review. J. Control. Rel. 65(1), 271-284 (2000).

12. Meng X, Chen $Y$, Li $Z$ et al. Nitrogen heterocyclic tryptamine ketone derivative and application as IDO and/or TDO inhibitor. WO2017173973A1 (2017).

13. Elise L, Claudie B, Patrick C. Nanoprecipitation and the "Ouzo effect": application to drug delivery devices. Adv. Drug Deliv. Rev. 71(2997), 86-97 (2014).

14. Zhu X, Sun J, Zhang Y et al. Immunization with functionalized carbon nanotubes enhances the antibody response against mode antigen ovalbumin. Immunol. Lett. 178, 77-84 (2016).

15. Shen Z, Reznikoff G, Dranoff G et al. Cloned dendritic cells can present exogenous antigens on both MHC class I and class II molecules. J. Immunol. 158(6), 2723-2730 (1997). 
16. Yue EW, Brent D, Brian W et al. Discovery of potent competitive inhibitors of indoleamine 2,3-dioxygenase with in vivo pharmacodynamic activity and efficacy in a mouse melanoma model. J. Med. Chem. 52(23), 7364-7367 (2009).

17. Stefani S, Spaapen RM, Yuanyuan Z et al. Up-regulation of PD-L1, IDO, and T(regs) in the melanoma tumor microenvironment is driven by CD8 ${ }^{+}$T cells. Sci. Transl. Med. 5(200), 200ra116 (2013).

18. Liu X, Niu S, Koblish HK et al. Selective inhibition of IDO1 effectively regulates mediators of antitumor immunity. Blood 115(17), 3520-3530 (2010).

19. Wang R, Tang A. Simultaneous determination of kynurenine and tryptophan in serum by high performance liquid chromatography. Se Pu 24(2), 140-143 (2006).

20. Fang Y, Lin Y, Su Y et al. Tryptanthrin-loaded nanoparticles for delivery into cultured human breast cancer cells, MCF7: the effects of solid lipid/liquid lipid ratios in the inner core. Chem. Pharm. Bull. (Tokyo). 59(2), 266-271 (2011).

21. Zhang Q, Wu H. Preparation and characterization of tryptanthrin-loaded nano-micelles. J. Northwest. Pharm. 31(3), 277-281 (2016).

22. Chen Y, Xia R, Huang Y et al. An immunostimulatory dual-functional nanocarrier that improves cancer immunochemotherapy. Nat. Commun. 7, 13443 (2016).

23. Chen Y, Sun J, Huang Y et al. Improved cancer immunochemotherapy via optimal co-delivery of chemotherapeutic and immunomodulatory agents. Mol. Pharm. 15(11), 5162-5173 (2018).

24. Feng B, Zhou F, Hou B et al. Binary cooperative prodrug nanoparticles improve immunotherapy by synergistically modulating immune tumor microenvironment. Adv. Mater. 30(38), e1803001 (2018).

25. Zhang G, Xing J, Wang Y et al. Discovery of novel inhibitors of indoleamine 2,3-dioxygenase 1 through structure-based virtual screening. Front. Pharmacol. 9, (2018).

26. Chen Y, Xia R, Huang Y et al. An immunostimulatory dual-functional nanocarrier that improves cancer immunochemotherapy. Nat. Commun. 7, 13443 (2016).

27. Jiang P, Zhao Y, Deng X et al. Antitumor and antimetastatic activities of chloroquine diphosphate in a murine model of breast cancer. Biomed. Pharmacother. 64(9), 609-614 (2010).

28. Wang J, Zhang C, Chia W et al. Haem-activated promiscuous targeting of artemisinin in Plasmodium falciparum. Nat. Commun. 6, 10111 (2015).

29. Coletti A, Greco FA, Dolciami D et al. Advances in indoleamine 2,3-dioxygenase 1 medicinal chemistry. MedChem Comm. 8(7), 1378-1392 (2017).

30. Sharma VM, Prasanna P, Seshu KVA et al. Novel indolo[2,1-]quinazoline analogues as cytostatic agents: synthesis, biological evaluation and structure-activity relationship. Bioorg. Med. Chem. Lett. 12(17), 2303-2307 (2002).

31. Wu Y, Xu T, Liu J et al. Structural insights into the binding mechanism of IDO1 with hydroxylamidine based inhibitor INCB14943. Biochem. Biophys. Res. Commun. 487(2), 339-343 (2017).

32. Yu S, Chern J, Chen T et al. Cytotoxicity and reversal of multidrug resistance by tryptanthrin-derived indoloquinazolines. Acta Pharmacol. Sin. 31(2), 259-264 (2010).

33. Yu S, Chen T, Tseng $S$ et al. Tryptanthrin inhibits MDR1 and reverses doxorubicin resistance in breast cancer cells. Biochem. Biophys. Res. Commun. 358(1), 79-84 (2007).

34. Onambele LA, Riepl H, Fischer R et al. Synthesis and evaluation of the antiplasmodial activity of tryptanthrin derivatives. Int. J. Parasitol. Drugs Drug Resist. 5(2), 48-57 (2015).

35. Iii DEO, Peppas NA. Opsonization, biodistribution, and pharmacokinetics of polymeric nanoparticles. Int. J. Pharm. 307(1), 93-102 (2006).

36. Davis ME, Zhuo C, Dong MS. Nanoparticle therapeutics: an emerging treatment modality for cancer. Nat. Rev. Drug Discov. 7(9), 771-782 (2008).

37. Wu J, Waxman DJ. Immunogenic chemotherapy: dose and schedule dependence and combination with immunotherapy. Cancer Lett. 419, 210-221 (2018).

- Inspired us to the potential cytotoxicity of CY-1-4 nanoparticles to immune cells.

38. Cairo G, Recalcati S, Mantovani A et al. Iron trafficking and metabolism in macrophages: contribution to the polarized phenotype. Trends Immunol. 32(6), 241-247 (2011).

39. Madeddu C, Gramignano G, Kotsonis P et al. Microenvironmental M1 tumor-associated macrophage polarization influences cancer-related anemia in advanced ovarian cancer: key role of Interleukin-6. Haematologica 103(9), e388-e391 (2018).

40. Deshmukh R, Trivedi V. Phagocytic uptake of oxidized heme polymer is highly cytotoxic to macrophages. PLoS ONE 9(7), e103706 (2014).

41. Rébé C, Ghiringhelli F. Cytotoxic effects of chemotherapy on cancer and immune cells: how can it be modulated to generate novel therapeutic strategies? Future Oncol. 11(19), 2645-2654 (2015).

42. Munn DH, Sharma MD, Lee JR et al. Potential regulatory function of human dendritic cells expressing indoleamine 2,3-dioxygenase. Science 297(5588), 1867-1870 (2002). 